Accelerator Division

Alternating Gradient Synchrotron Department BROOKHAVEN NATIONAL LABORATORY

Upton, New York 11973

Accelerator Division

Technical Note

AGS/AD/Tech. Note No. 391

\title{
STOPBAND CORRECTION OF THE AGS BOOSTER -- SUMMARY OF CORRECTION PARAMETERS AND HARMONIC ANALYSIS OF IMPERFECTIONS
}

Y. Shoji* and C.J. Gardner

March 16, 1994

*Visitor from KEK, Japan. 


\section{Stop-band Correction of the AGS Booster ---- \\ Summary of Correction Parameters and Harmonic Analysis of Imperfections}

Y. Shoji and C. J. Gardner

\section{ABSTRACT}

The harmonic components of magnetic field imperfections in the AGS Booster has been determined through careful measurements of the field corrections required to compensate imperfections which drive various transverse resonances. An analysis of the required correction yielded phase and amplitude information which points to possible locations and strength of field imperfections, and the dependence of these corrections on the bending field (B), dB/dt, and the mean closed orbit radius (dR) suggested possible sources of imperfections. In particular the dependence of the required correction on $\mathrm{dB} / \mathrm{dt}$ strongly suggested an error of the windings on the dipole vacuum chambers which compensate quadrupole and sextupole fields produced by the eddy-currents. The dependence of the required quadrupole and the sextupole correction on $d R$ indicated the presence of sextupole and octupole imperfections of the same harmonics. The observations also suggested the presence of a strong harmonic remnant field. 
contents

\section{ABSTRACT}

I INTRODUCTION

II MEASUREMENT AND PARAMETERS

III OVERVIEW OF THE RESULTS

IV B TERM

IV-1 C.O.D. and down-feeding

IV-2 Normal quadrupole imperfection

IV-3 Skew quadrupole imperfection

IV-4 Sextupole imperfection

IV-5 Octupole imperfection

$V \quad \mathrm{~dB} / \mathrm{dt}$ TERM

V $V-1 \quad$ Dipole imperfection

$V-2$ Normal quadrupole imperfection

V-3 Normal sextupole imperfection

$\mathrm{V}-4 \quad$ Other imperfections

VI OFF-SET TERM

VII DISCUSSIONS

VII-I Estimation of imperfections

VII-2 Improvement of correction accuracy

VII-3 other comments

ACKNOWLEDGEMENT

Appendix I Magnet production errors

Appendix II Calculation memo about the correction parameter unit

Appendix III Expected correlation of two resonances

Appendix IV Chromaticity of the AGS Booster

Appendix V Eddy-current field correction at C5 
Stop-band (transverse resonance) correction is one of the most essential parts of high intensity proton synchrotron. Because of space-charge tune spread protons encounter many resonances especially at injection and early acceleration. The AGS Booster will have a tune spread as large as 0.5 at the design intensity of $1.5 \times 10^{12} \mathrm{ppp}$. Up to the 3 rd order resonances should be corrected in the working space: $4.0<Q x, Q Y<5.0$ for a high intensity and low emittance operation.

This year we improved our knowledge about the stop-band correction of the Booster through many kinds of machine studies. [1-19]. The most important change was brought in by the skew sextupole correction strings [11], which were not initially build in [20-23]. However it was not strange that the Booster needed the skew sextupole correction $[20,24,25]$. It enabled us to inject the design intensity into the Booster $[26,27]$. The second was two kinds of down-feeding effect. The first kind was produced by a systematic sextupole field and C.O.D. (orbit imperfection). It showed us a necessity of a precise control of C.O.D. [8]. The second kind was produced by a sextupole harmonic imperfection and a dispersion (systematic displacement), which had not been considered before. This effect appeared as a linear dependence of correction strength on mean radius: dR (or momentum displacement). Then we usualiy refer it 'slope'. We succeeded to cancel this slope by the additional sextupole correction string $[9,10,14,17,21]$. The fine tune space survey was the other important result. It confirmed the correction of stop-bands [12]. The measurement of its intensity dependence proved the large space charge tune shift $[13,16]$. In addition to these we parameterized the correction strength of each resonance $[2-5,11,14]$. We separated the correction fields into three terms; 1) remnant field (which did not depend on $B$ neither $\mathrm{dB} / \mathrm{dt}$, referred as off-set term), 2) misalignment or imperfection of magnets (which was proportional to B, referred as B term) and 3) eddy-current imperfection (which was proportional to $\mathrm{dB} / \mathrm{dt}$, referred as $\mathrm{dB} / \mathrm{dt}$ term). That parameterization enables us to apply resonance correction to an arbitrary magnet cycle. These parameters are basic information to understand the imperfection of the Booster. For example, Y.Y. Lee could indicate the misconnection of the eddy-current correction winding from a stop-band data.

In this report we will discuss about the last subject of the stop-band studies: imperfection analysis. In section II we will show the method we used to measure the strengths of stop-bands and the definitions of parameters. In section III we will show the summary table of the correction parameters. A discussion about the reliability and the data cross check will be given in the same section. From section IV to section VI we will try to explain the observed imperfections from origins of imperfections. In section VII we will summarize and discuss about some problems of correcting stop-bands. There has been some estimations of the ring 
imperfections before the ring was constructed and now we will revise the estimations using the data of field measurements of the real magnets and will check the imperfections measured through the stop-band studies. The results of the field measurement are reprinted in Appendix I [28-33] for a convenjence of readers. The comparison of the measured imperfection with the present estimation and the estimations done before will be valuable for whom wants to design and construct new synchrotron. 


\section{MEASUREMENT AND PARAMETERS}

Resonances, except for $Q \mathrm{Qx}-\mathrm{QY}=0$, were observed by programming the tunes to pass through each resonance at various timings during the magnet cycle (at various $B$ and dB/dt). Fig.l shows an example of measured points in the magnet cycle. The amount of the beam loss by the resonance crossing was measured at several different correction settings in order to determine a setting which minimized the loss. Fig.2 shows an example of plots to determine the correction values [34]. A curve was not always parabolic and sometimes was not symmetric and a curve of a weak resonance had a flat bottom. Then the eye-ball found a best point. The correction strength of $Q x-Q Y=0$ was measured by $W$. van Asselt. He adjusted the skew quadrupole components to decouple the horizontal and the vertical betatron oscillations $[35,27]$.

The correction values were fitted with the following function:

$$
N(\mathrm{xxxx})=\mathrm{Co}+\mathrm{Cb} B+\mathrm{Cbt}(\mathrm{dB} / \mathrm{dt}) \cdot(\mathrm{II}-1)
$$

Here $N(x x x x)$ is the strength of correction which unit was selected for a convenience of computer control. Their definitions are given in the reference [23] and Appendix II of this Tech. Note. The $x x x x$ in the bracket will be replaced by a harmonic number and an order of resonance. $\mathrm{Co}, \mathrm{Cb}$ and $\mathrm{Cbt}$ are fitting parameters which correspond to off-set term (remnant field imperfection), B term (misalignment and magnet production error) and dB/dt term (eddycurrent imperfection), respectively. $B$ is the field strength of the main bending dipoles connected with the momentum (P) by

ox

$$
B(k G)=2 \pi(10 /(36 \times 2.42)) P=0.721 \mathrm{P}(\mathrm{Tm}) \quad(I I-2 \mathrm{a})
$$

$$
\mathrm{B}(\mathrm{kG})=2.405 \mathrm{P}(\mathrm{GeV} / \mathrm{C}) \quad \text { (II-2b) }
$$

The unit of $B$ and $d B / d t$ are $k G$ and $\mathrm{G} / \mathrm{ms}=\mathrm{kG} / \mathrm{s}$, respectively.

The correction $\delta N(x \times x x) / \delta d R s e t$ presents the slope, which is the dependence of $\mathrm{N}(\mathrm{x} \times \mathrm{xx})$ on the set value of the radial steering parameter (dRset). The parameter dRset is connected with the momentum displacement $(\mathrm{dP} / \mathrm{P})$ by the following equation [2]:

$$
\operatorname{dRset}(\mathrm{cm})=319(\mathrm{dP} / \mathrm{P}) \text {. }
$$

At the present (Nov.1993) correction strings: SH3, SV3, SH4 and SV4 are controlled with different names, such as

$$
\begin{aligned}
& \mathrm{N}(\cos 14 \mathrm{X})=20 * \mathrm{SV} 3, \quad \mathrm{~N}(\sin 14 \mathrm{X})=20 * \mathrm{SH} 3, \\
& N(\cos 14 X Y)=20 * S V 4 \text { and } N(\sin 14 X Y)=20 * S H 4 \text {, }
\end{aligned}
$$

but their names are not appropriate and are expected to be changed. So in this report we use the original names of power supplies: SH3, SV3, SH4 and SV4. Their unit is a current in Ampere of each power supply. 
$N(x \times 9 X)$ represents both $N(\cos 9 x)$ and $N(\sin 9 x)$, and $N(9 x)$ presents the amplitude of correction:

$$
N(9 X)=\sqrt{ }\left[N(\cos 9 x)^{2}+N(\sin 9 x)^{2}\right],
$$

and vice versa.

The definition of integrated multipole field components (A0, $\mathrm{B} 0, \mathrm{~A} 1, \mathrm{~B} 1, \mathrm{~A} 2, \mathrm{~B} 2, \ldots-$ - follows the standard used by E.Blesser and $R$. Thern [28-31].

$$
\begin{aligned}
& \int_{s}^{s+\Delta s} B x[y=0] d s=A 0+A 1 x+A 2 x^{2}+A 3 x^{3}+---\quad \text { (II-5a) } \\
& \left.\int_{s}^{s+\Delta s} B y=0\right] d s=B 0+B 1 x+B 2 x^{2}+B 3 x^{3}+-\cdots(\text { II }-5 b)
\end{aligned}
$$

The unit of $A n$ and $B n$ is Tesla per meter ${ }^{n-1}\left(T / m^{n-1}\right)$. 


\section{OVERVIEW OF THE RESULTS}

The results of fittings with equation (II-1) are summarized and listed in Table $I$, which had been reported in the listed study reports. The values are a little bit different from the values reported at the IEEE Particle Accelerator Conference in 1993 [27], because they were reanalyzed considering errors of data points after the conference. The errors in Table I are different from the original study reports because they were normalized with reduced chai-square (multiplied by $\sqrt{ }\left(\chi^{2} / f\right)$ ) of the fitting when $\chi^{2} / f$ was larger than 1. The correction functions for the high intensity proton operation were calculated from these parameters and are shown in Fig.3. The correction functions for the $\mathrm{Au}^{+33}$ operation were also calculated and are shown in Fig.4.

Typical strengths of each correction term at near the proton injection $(B=2 \mathrm{kG}, \mathrm{dB} / \mathrm{dt}=50 \mathrm{kG} / \mathrm{ms}, \mathrm{dP} / \mathrm{P}= \pm 0.4)$ were calculated and are listed in Table II. Except the special case (dB/dt term of the skew quadrupole correction was negligibly small) non of three parameters $\mathrm{Co}, \mathrm{Cb}$ and $\mathrm{Cbt}$ were negligible. The residual stop-band produced by the 'slope' was also considerable.

The phase and amplitude of correction parameters of the 9 th and the 14th harmonic imperfections are shown in Fig.5. Any pair of resonances produced by the same imperfection are plotted in the same plane. The pairs are $2 Q x=9$ and $20 y=9$ produced by the 9 th normal quadrupole imperfection, $3 \mathrm{Qx}=14$ and $Q \mathrm{X}+2 \mathrm{QY}=14$ produced by the 14 th sextupole imperfection and the slopes of $2 \mathrm{QX}=9$ and $2 \mathrm{QY}=9$ produced by the 9 th normal sextupole imperfection. They are expected to have a correlation as is explained in Appendix III. The observed correlations were roughly in the expected range. The only one exception was the sine component of the off-set term of the 14th normal sextupole imperfection. We have no idea how to understand this results.

The absolute correction field strength are listed in Table III. The strength of the 1-th down-feeding to the resonance $\mathrm{mQx}+\mathrm{nQy}=\mathrm{k}$ was calculated as:

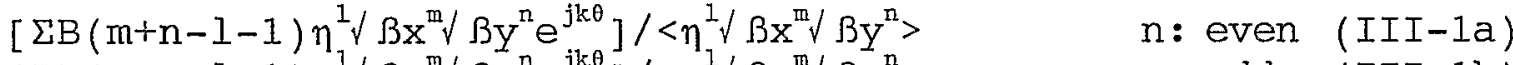

$$
\begin{aligned}
& {\left[\Sigma A(m+n-1-1) \eta^{1} / \beta x^{m} / \beta y^{n} e^{j k \theta}\right] /\left\langle\eta^{1} / \beta x^{m} / \beta y^{n}\right\rangle \quad n: \text { odd. (III-1b) }}
\end{aligned}
$$

Here $\eta, \beta x, \beta y$ and $\theta$ are dispersion, horizontal beta function, vertical beta function and harmonic phase. The bracket < > means an average through the ring (not only at the locations of correction elements). The harmonic phase $\theta$ for the resonance $n Q x+n Q y=n+m$ was simplified as

$$
(n+m) \theta=n(\mu x / Q x)+m(\mu y / Q Y) .
$$

Here $\mu \mathrm{x}$ and $\mu \mathrm{y}$ are horizontal and vertical betatron phase advance from the start point of the super period $A(s=0)$. 


$$
\mu x=\int_{0}^{s} \mathrm{ds} / \beta \mathrm{x} \text { and } \quad \mu \mathrm{y}=\int_{0}^{\mathrm{s}} \mathrm{ds} / \beta \mathrm{x} .
$$

Mainly we will use values calculated by A. Luccio and M.Blaskiewicz using the simulation code $\mathrm{MAD}$ at $Q \mathrm{QX}=4.633676$ and $\mathrm{QY}=4.583271$ [36].

The strength of the imperfection field of the normal sextupole field and the skew sextupole field were in the same order except $\mathrm{dB} / \mathrm{dt}$ terms. We have no reason that we need normal sextupole correction but we do not need skew sextupole correction. Because the magnets were well made and the magnetic field errors of the normal and the skew sextupole components were roughly the same (Table A-I - A-IV in Appendix I). The only one exception, the existence of the large normal sextupole $\mathrm{dB} / \mathrm{dt}$ term, suggested the imperfection of the eddy-current sextupole correction windings $[33,37,38,39]$. It was natural that we needed the skew sextupole corrections in addition to the normal sextupole corrections.

The magnitude of the sextupole error field estimated from the sextupole resonances and that from the 'slope' of quadrupole resonances were roughly the same. It meant that the same sextupole imperfection produced the sextupole stop-bands and the slope of the quadrupole stop-bands. Then in any other synchrotron if it requires a third resonance corrections, it should require the correction of quadrupole 'slope' to correct a half-integer resonance. 
Table I Stop-band correction parameters.

\begin{tabular}{|c|c|c|c|c|c|}
\hline $\begin{array}{l}\text { imperfection } \\
\text { resonance }\end{array}$ & $\begin{array}{l}\text { field } \\
\text { string }\end{array}$ & $\mathrm{Co}$ & $\begin{array}{c}\mathrm{Cb} \\
(/ \mathrm{kG})\end{array}$ & $\begin{array}{c}\mathrm{Cbt} \\
(/(\mathrm{G} / \mathrm{s}))\end{array}$ & rence \\
\hline \multicolumn{6}{|c|}{ normal quadrupole field } \\
\hline \multirow[t]{2}{*}{$2 Q x=9$} & $N(\cos 9 x)$ & $33 \pm 130$ & $101 \pm 31$ & $5.5 \pm 3.8$ & {$[3]$} \\
\hline & $N(\sin 9 x)$ & $-12 \pm 70$ & $122 \pm 64$ & $-1.5 \pm 1.3$ & \\
\hline \multirow[t]{2}{*}{$2 Q y=9$} & $N(\cos 9 Y)$ & $138 \pm 18$ & $91 \pm 7$ & $3.36 \pm 0.11$ & {$[4]$} \\
\hline & $\mathrm{N}(\sin 9 \mathrm{Y})$ & $-43 \pm 26$ & $39 \pm 9$ & $-6.30 \pm 0.20$ & \\
\hline \multicolumn{6}{|c|}{ skew quadrupole field } \\
\hline$Q x-Q y=0$ & $N(\cos 0 X Y)$ & -180 & 140 & 0 & {$[27]$} \\
\hline \multirow[t]{2}{*}{$\tilde{Q} x+Q y=9$} & $N(\cos 9 X Y)$ & $35 \pm 55$ & $49.2 \pm 7.2$ & $0.04 \pm 0.53$ & {$[5]$} \\
\hline & $N(\sin 9 \times Y)$ & $-111 \pm 45$ & $28.5 \pm 6.0$ & $-0.11 \pm 0.41$ & \\
\hline \multicolumn{6}{|c|}{ normal sextupole field } \\
\hline \multirow[t]{2}{*}{$3 Q x=14$} & $N(\cos 14 X)$ & $48 \pm 70$ & $-31 \pm 34$ & $3.49 \pm 0.43$ & [2] \\
\hline & $N(\sin 14 x)$ & $-129 \pm 34$ & $40 \pm 16$ & $6.00 \pm 0.20$ & \\
\hline \multirow[t]{2}{*}{$Q x+2 Q y=14$} & $\mathrm{~N}(\cos 14 \mathrm{XY})$ & $5 \pm 29$ & $14 \pm 11$ & $4.74 \pm 0.20$ & [2] \\
\hline & $N(\sin 14 X Y)$ & $-103 \pm 24$ & $17 \pm 9$ & $2.64 \pm 0.19$ & \\
\hline \multirow{2}{*}{\multicolumn{6}{|c|}{$Q x+2 Q y=13$}} \\
\hline & & & & & \\
\hline \multirow[t]{2}{*}{$2 Q x=9$} & $\mathrm{SV} 3 * 20$ & $90 \pm 160$ & $67 \pm 61$ & $-0.07 \pm 0.51$ & {$[17]$} \\
\hline & $\mathrm{SH} 3 * 20$ & $250 \pm 110$ & $8 \pm 33$ & $3.15 \pm 0.87$ & \\
\hline \multicolumn{2}{|c|}{$\delta N(\cos 9 x) / \delta d R s e t$} & t $\quad 75 \pm 40$ & $-3 \pm 12$ & $1.06 \pm 0.29$ & {$[17]$} \\
\hline \multicolumn{2}{|c|}{$\delta N(\sin 9 x) / \delta d R s e t$} & $52 \pm 40$ & $13 \pm 12$ & $0.45 \pm 0.29$ & \\
\hline \multirow[t]{2}{*}{$2 Q Y=9$} & $\mathrm{SV} 3 * 20$ & $69 \pm 90$ & $8 \pm 28$ & $-4.06 \pm 1.01$ & {$[14]$} \\
\hline & $\mathrm{SH} 3 * 20$ & $270 \pm 154$ & $123 \pm 70$ & $13.35 \pm 0.64$ & \\
\hline \multicolumn{2}{|c|}{$\delta \mathrm{N}(\cos 9 \mathrm{Y}) / \delta \mathrm{dRs}$. } & t $\quad 49 \pm 25$ & $21 \pm 9$ & $0.94 \pm 0.18$ & [14] \\
\hline \multicolumn{2}{|c|}{$\delta N(\sin 9 Y) / \delta d R s e t$} & $-22 \pm 9$ & $1 \pm 3$ & $-0.44 \pm 0.06$ & \\
\hline \multicolumn{6}{|c|}{ skew sextupole field } \\
\hline \multicolumn{3}{|c|}{$3 \mathrm{QY}=14$} & unable to & parameterize & [11] \\
\hline \multirow[t]{2}{*}{$2 Q x+Q Y=14$} & $\mathrm{SV} 4 * 20$ & $720 \pm 120$ & $-152 \pm 42$ & $6.82 \pm 0.70$ & $[1]]$ \\
\hline & $\mathrm{SH} 4 * 20$ & $604 \pm 81$ & $30 \pm 30$ & $-0.30 \pm 0.64$ & \\
\hline \multirow{2}{*}{\multicolumn{6}{|c|}{$\begin{array}{l}3 Q y=13 \\
20 x+0 y=13\end{array}$}} \\
\hline \multirow{2}{*}{\multicolumn{6}{|c|}{$\begin{array}{l}2 Q x+Q y=13 \\
O x-O y=0\end{array}$}} \\
\hline & & $\perp$ & & & \\
\hline \multicolumn{6}{|l|}{$Q x+Q y=9$} \\
\hline \multirow{2}{*}{$\delta N$} & os $9 X Y) / \delta d R s \in$ & set $-19.9 \pm 1$ & $0-0.4 \pm 0.6$ & $0.024 \pm 0.03$ & {$[17]$} \\
\hline & in $9 x Y) / .8 d R s \epsilon$ & set $9.8 \pm 1$ & $01.6 \pm 0.6$ & $0.044 \pm 0.03$ & \\
\hline \multicolumn{6}{|c|}{ normal octupole field } \\
\hline \multirow[t]{2}{*}{$2 Q x=9$} & $\delta N(\cos 9 X) / \varepsilon$ & $\delta\left(\right.$ dRset $\left.^{2}\right)$ & $-13 \pm 7$ & ) & [19] \\
\hline & $\delta N(\sin 9 x) / \delta$ & $\delta\left(\right.$ dRset $\left.^{2}\right)$ & $10 \pm 7$ & & \\
\hline $2 Q y=9$ & $\delta N(\cos 9 Y) / 8$ & $\delta\left(\right.$ dRset $\left.^{2}\right)$ & $-10 \pm 9$ & ) & [19] \\
\hline & $\delta N(\sin 9 Y) / \delta$ & $\delta\left(\right.$ dRset $\left.^{2}\right)$ & $15 \pm 9)$ & & \\
\hline $3 Q x=14$ & $\delta N(\cos 14 X)$ & / SdRset & $(69)$ & & [2] \\
\hline & $\delta N(\sin 14 x)$ & / dRset & $(-63)$ & & \\
\hline
\end{tabular}


Table II Relative strengths of the correction terms. The units were arbitrary selected for a convenience of computer control. They were calculated at $B=2 \mathrm{kG}, \mathrm{dB} / \mathrm{dt}=50 \mathrm{G} / \mathrm{ms}$ and $\mathrm{dP} / \mathrm{P}= \pm 0.4 \%$. Correction currents of the $3 Q \mathrm{y}=14$ and the slope of $3 \mathrm{Qx}=14$ were measured only at $\mathrm{B}=1.7 \mathrm{kG}$ and $\mathrm{dB} / \mathrm{d} t=0$. Their results are listed in the last column $(\mathrm{N}(\mathrm{x} \times \mathrm{xx}))$.

\begin{tabular}{|c|c|c|c|c|c|}
\hline resonance & & $\mathrm{CO}$ & $\begin{array}{c}\mathrm{Cb} \\
\mathrm{a} 2 \mathrm{kG}\end{array}$ & $\begin{array}{l}\mathrm{Cbt} \\
\text { @ } 50 \mathrm{G} / \mathrm{ms}\end{array}$ & $N(x \times x x)$ \\
\hline \multirow[t]{2}{*}{$2 Q x=9$} & $N(9 X)$ & $<180$ & 380 & 280 & \multirow{4}{*}{13} \\
\hline & $\begin{array}{l}\delta N(9 X) / d R s e t \\
\delta^{2} N(9 X) / d_{R s e t} 2\end{array}$ & 120 & $<50$ & 70 & \\
\hline \multirow[t]{2}{*}{$2 Q Y=9$} & $N(9 Y)$ & 150 & 200 & 360 & \\
\hline & $\begin{array}{l}\delta N(9 Y) / d R s e t \\
\delta^{2} N(9 Y) / d^{2} s e{ }^{2}\end{array}$ & 70 & 50 & 70 & \\
\hline$Q x-Q Y=0$ & $\mathrm{~N}(\cos 0 \mathrm{XY})$ & 180 & 280 & 0 & \multirow{2}{*}{14} \\
\hline$Q x+Q Y=9$ & $\begin{array}{l}N(9 X Y) \\
\delta N(9 X Y) / \text { dRset }\end{array}$ & $\begin{array}{r}120 \\
28\end{array}$ & $\begin{array}{r}110 \\
4\end{array}$ & $\begin{array}{r}<30 \\
3\end{array}$ & \\
\hline $3 Q x=14$ & $\begin{array}{l}N(14 X) \\
\delta N(14 X) / d R s e t\end{array}$ & 150 & 100 & 350 & \multirow{2}{*}{120} \\
\hline$Q x+2 Q y=14$ & $N(14 X Y)$ & 300 & 150 & 160 & \\
\hline $3 Q Y=14$ & $\mathrm{SH} 4, \mathrm{SV} 4$ & & & & \multirow[t]{2}{*}{$>70$} \\
\hline $2 Q X+Q Y=14$ & $\mathrm{SH} 4, \mathrm{SV} 4$ & 50 & 16 & 17 & \\
\hline
\end{tabular}


Table III Averaged error field strengths. The strength of the l-th down-feeding to the resonance mQx+nQy=k was defined as equation (III-1). The strength of octupole imperfection were observed only at $1.7 \mathrm{kG}$ flat porch ( $\mathrm{dB} / \mathrm{dt}=0$ ). So the observed strength were sum of off-set terms and $B$ terms. The listed values in the brackets were values calculated under the assumption that they came from only the off-set term or the B term.

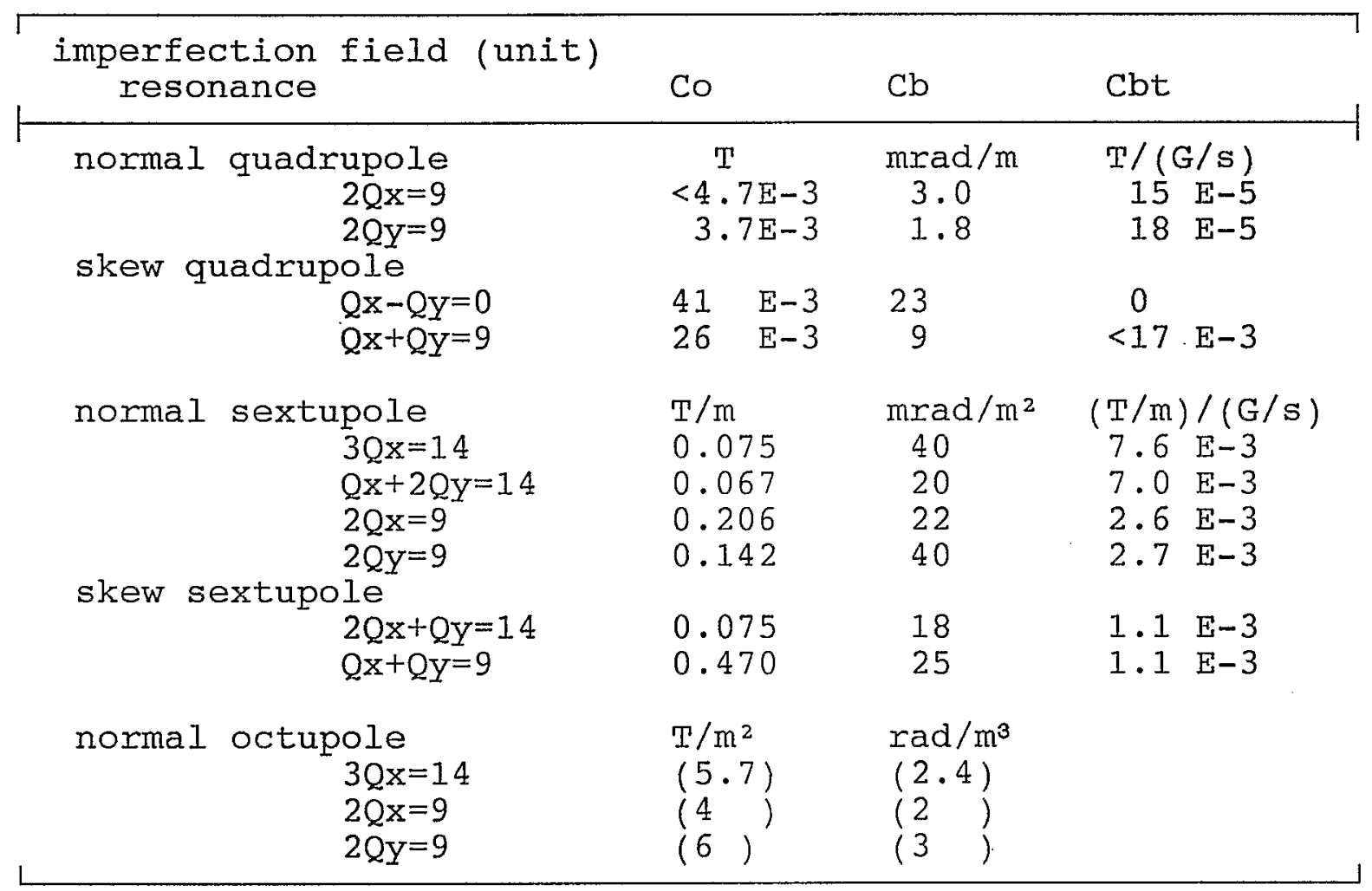


IV B TERM

IV-1 C.O.D. and down-feeding

Harmonic components of horizontal and vertical C.O.D. are necessary information to analyze the imperfection of the ring. The first kind of down-feeding effect produces lower order stop-bands. The harmonic components of C.O.D. Of the AGS Booster were measured by $K$. Brown et al [40] and by others [8,41-43]. We discussed about this subject in SR-307 [18]. The horizontal and the vertical C.O.D. were mainly produced by a random misalignment of elements. The amplitudes of harmonic components of the horizontal and the vertical C.O.D. were about $1 \mathrm{~mm}$ and $0.3 \mathrm{~mm}$, respectively. In this report we will use these values as the dipole error source.

IV-2 Normal quadrupole imperfection

Ninth half-integer stop-band width is calculated by the following equation:

$$
d Q=(1 / 2 \pi) \mid \int_{0}^{2 \pi R} \delta K_{1} \text { Berr } e^{j 9 \theta} d s \mid \text {. }
$$

When $\mathrm{N}$ dipole errors are random and they locate at the same $\beta=\beta e r r$, the r.m.s. of expected stop-band width $\sqrt{ }\left\langle\mathrm{dQ}^{2}\right\rangle$, which is also a statistically mostly expected stop-band width, is

$$
\sqrt{ }\left\langle\mathrm{dQ}^{2}\right\rangle=(1 / 2 \pi) \text { Berr } \sqrt{ } \operatorname{Nerr}\left(\delta \mathrm{K}_{1} \mathrm{ds}\right) \text { rms . }
$$

The stop-band widths produced by the random magnet imperfections were calculated using the above equation. The results are listed in Table IV. The random quadrupole imperfection of the dipole magnets was larger than that of the quadrupole magnets.

Half-integer stop-band widths produced by the first kind of down-feeding (C.O.D. at the sextupole field) was estimated. The known systematic sextupole fields in the ring were edge sextupole of the bending dipoles and chromaticity control sextupoles. The strength of the random sextupoles were negligibly small (Appendix IV). The edge sextupoles were measured by R.Thern [28] or G.Danby and J.Jackson [32]. Typical strengths of the chromaticity sextupoles were calculated in Appendix IV and used in this estimation of the imperfection. When there are 9 th harmonic C.O.D. at the periodic sextupoles a half-integer stop-band width produced by this imperfection was

dQ

$$
\begin{aligned}
& =(1 / 2 \pi) \quad \Sigma \text { Berr } 2(B 2 / B \rho) \delta X \\
& =(1 / 2 \pi) \text { Nerr Berr 2 (B2/B } \rho) \text { Xo }(2 / \pi) .
\end{aligned}
$$


Here Nerr and Berr are number of error elements and beta function at the sextupoles, respectively. Xo is the 9 th harmonic amplitude of C.O.D. (this is not the rms) and

$$
\begin{aligned}
& \delta x=X O \cos (n \theta+\theta 0), \\
& (2 / \pi)=\langle|\cos (n \theta+\theta 0)|\rangle .
\end{aligned}
$$

The stop-band widths were calculated using the above equation (IV$3)$. Here we calculated only the coupling of the 9 th harmonic C.O.D. and the 0th harmonic sextupole component and ignored the other couplings such as the 3rd harmonic C.O.D. and the 6th harmonic sextupole, the 3 rd harmonic C.O.D. and the 12 th harmonic sextupole, the 9th harmonic C.O.D. and the 18th harmonic sextupole, etc. because their contributions were small. The results and parameter values are listed in Table $V$. The estimated stop-band widths are the sum of the results of Table IV and Table V, which are

$$
\mathrm{dQx}=0.0035 \text { and } \mathrm{dQy}=0.0031 \text {. }
$$

Here dQX and dQY are the stop-band widths of $2 Q x=9$ and $2 Q y=9$, respectively. These stop-bands mainly came from the displacement at the edges of the bending dipoles (the first kind of down-feeding). The contributions from the magnet imperfections were rather small.

On the other hand the observed stop-band widths produced by the $B$ terms were

$$
\begin{aligned}
& \mathrm{dQX}=\sqrt{ }\left(101^{2}+122^{2}\right) \times 10^{-5} \times 2.405 \\
& \mathrm{dQY}=\sqrt{ }\left(91^{2}+39^{2}\right) \times 10^{-5} \times 2.405
\end{aligned}
$$

\begin{tabular}{|c|c|c|c|c|c|c|}
\hline magnet & Nerr & $\begin{array}{l}<\beta x> \\
(m)\end{array}$ & $\begin{array}{l}<\beta y> \\
(\mathrm{m})\end{array}$ & $\begin{array}{l}\left(\delta \mathrm{K}_{1} \mathrm{ds}\right) \mathrm{rms} \\
(\mathrm{rad} . / \mathrm{m})\end{array}$ & $\begin{array}{c}d Q x \\
(E-5)\end{array}$ & $\begin{array}{l}d Q y \\
(E-5)\end{array}$ \\
\hline B & 36 & 8.6 & 8.7 & $2 \pi / 36 \times 9.1 \mathrm{E}-4$ & 130 & 130 \\
\hline $\mathrm{QF}$ & 24 & 13.4 & 4.1 & $0.270 \times 2.6 \mathrm{E}-4$ & 80 & 80 \\
\hline$Q D$ & 24 & 4.0 & 13.6 & $0.276 \times 2.6 \mathrm{E}-4$ & & J \\
\hline tota] & & & & & 150 & 150 \\
\hline
\end{tabular}

They agreed very well with the estimations.

Table IV Estimation of the half-integer stop-band widths produced by the imperfection of magnets. 
Table V Estimation of the half-integer stop-band widths down-fed from the sextupole field.

\begin{tabular}{|c|c|c|c|c|c|c|c|}
\hline magnet & Nerr & $\begin{array}{r}<\beta x> \\
(m)\end{array}$ & $\begin{array}{r}<B x> \\
(m)\end{array}$ & $\begin{array}{l}\mathrm{B} 2 / \mathrm{Bp} \\
(/ \mathrm{m})\end{array}$ & $\begin{array}{c}\text { Xo } \\
\text { (mm) }\end{array}$ & $\begin{array}{l}d Q x \\
(\mathrm{E}-5\end{array}$ & $\begin{array}{l}d Q y \\
E-5)\end{array}$ \\
\hline $\begin{array}{l}\text { B edge } \\
\text { SexF } \\
\text { SexD }\end{array}$ & $\begin{array}{l}72 \\
24 \\
24\end{array}$ & $\begin{array}{r}8.6 \\
12.0 \\
4.5\end{array}$ & $\begin{array}{r}8.7 \\
4.6 \\
12.3\end{array}$ & $\begin{array}{c}2 \pi / 72 \times 0.24 \\
0.053 \\
0.003\end{array}$ & $\begin{array}{l}1.1 \\
0.6 \\
1.0\end{array}$ & $\begin{array}{r}260 \\
190 \\
10\end{array}$ & $\begin{array}{r}260 \\
70 \\
20\end{array}$ \\
\hline total & & & & & & 320 & 270 \\
\hline
\end{tabular}

IV-3 Skew quadrupole imperfection

The strength of the skew quadrupole imperfections were larger than those of the normal quadrupole imperfections. When we define stop-band width with the same way as the normal quadrupole imperfections:

$$
\begin{aligned}
& \operatorname{dQxy} y_{-}=(1 / 2 \pi)||_{0}^{2 \pi R} \delta K_{1}, \sqrt{ } \beta x / \beta y d s \mid \text { and } \\
& d Q x y_{+}=\left.(1 / 2 \pi)\right|_{0} ^{2 \pi R} \delta K_{1} / \sqrt{ } \beta x / \beta y e^{j 9 \theta} d s \mid \text {. }
\end{aligned}
$$

Here dQxy and dQxy+ are the widths of $Q x-Q Y=0$ and $Q x+Q Y=9$, respectively. The $\delta K_{1}^{\prime}$ is

$$
\int_{s}^{s+\Delta s} \delta K_{1}^{\prime} d s=A 1 / B p .
$$

Their measured stop-band widths were

$$
\begin{aligned}
& \mathrm{dQXY}_{-}=140\left(4 \times 10^{-5} \times 2.405\right) \quad=0.0135 \text { and }
\end{aligned}
$$

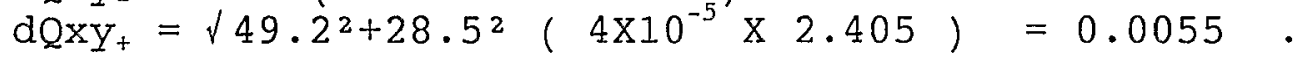

These were larger than the stop-band widths of the normal quadrupole imperfections.

On the other hand the estimated stop-band widths using the equations (IV-2) and (IV-3) are listed in Table VI, which are

$$
\mathrm{dQxy}_{-} \quad=0.0013 \text { and dQxy+ }=0.0012 \text {. }
$$

These are much smaller than the estimations of the normal 
quadrupole imperfections because the contributions from the downfeeding were smaller. In this estimation the used random rotation error of quadrupole magnets were 0.3 mrad. rms, which was the designed accuracy of the alignment [44]. The observed stop-band widths were 5 - 10 times larger than the estimations.

We have no data to explain this difference. One possibility was a longitudinal magnetic field to which the magnetic field measurement was blind. The other possibility was a misestimation of rotation of the quadrupole magnet. We have a reason to suspect that the alignment was worse than we expected. The observed amplitude of the vertical C.O.D. gave us a limit of the random rotation error of bending dipoles (not of the quadrupole). The rms rotation error should be considerably smaller than 5 mrad. [18]. Then we can not reject the possibility of a large rotation error. The measurement suggested that the systematic skew component (dQXY-) were about the twice of the random skew component (dQxy+). We should also check the control program because it could have a bug [17].

Table VI Estimation of the skew quadrupole stop-band width.

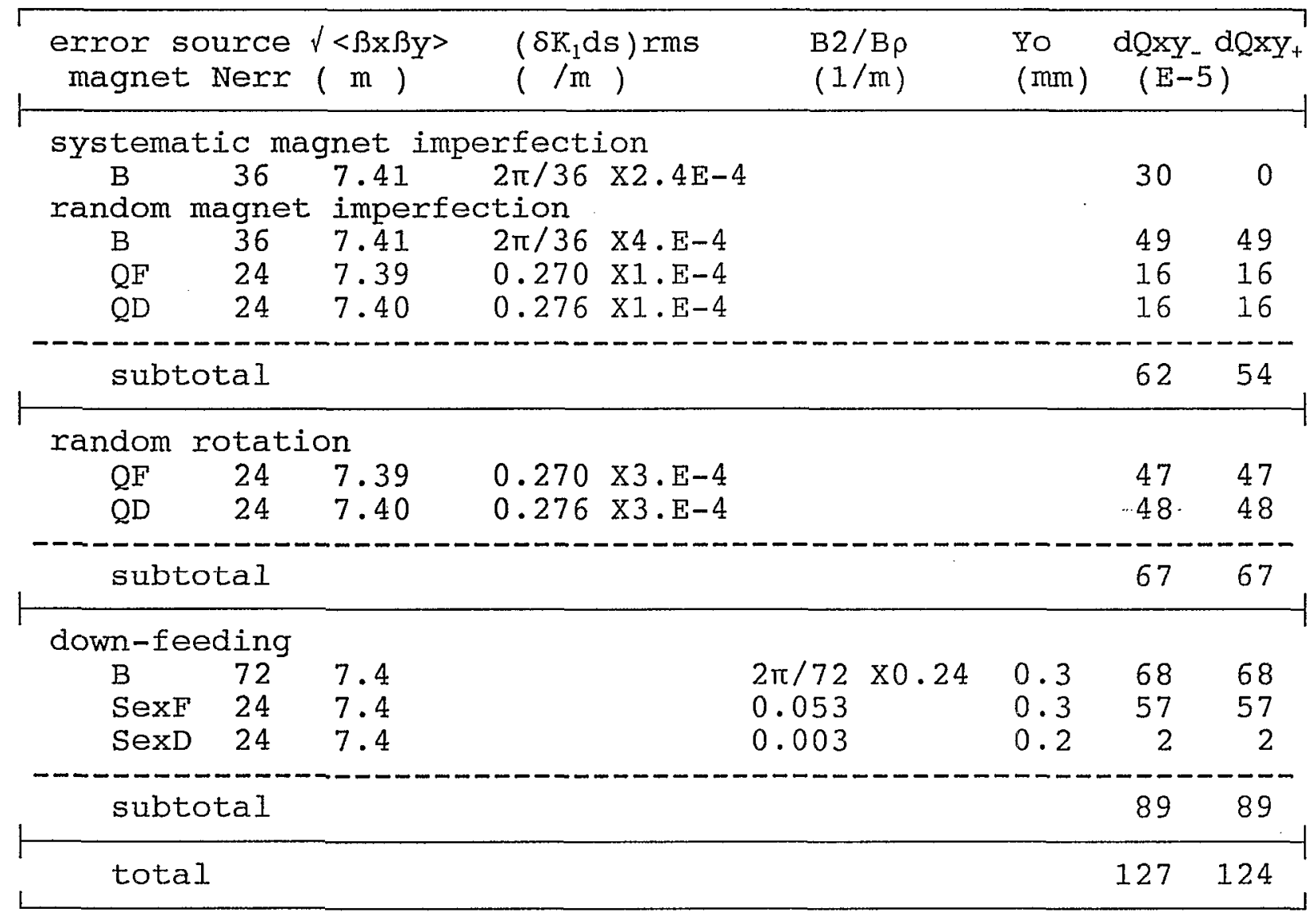


IV-4 Sextupole Imperfection

The strength of the normal and the skew sextupole imperfections were measured as the strength of $3 r d$ resonance corrections and also the down-feeding to the 2 nd resonances. Then we will calculate the averaged strength of harmonic sextupole imperfections to compare the expected values with the observed, because the stop-band width can not be a common scale. The observed strengths of the sextupole imperfections are listed in Table VII. The strength of the imperfections were $20-40 \mathrm{rad} . / \mathrm{m}^{2}$.

When the rms of Nerr random sextupoles of magnets is $\left(\delta \mathrm{K}_{2} \mathrm{ds}\right) \mathrm{rms}$ the mostly expected strength of harmonic sextupole imperfection dSext is

$$
\text { dSext }=\sqrt{ } \operatorname{Nerr}\left(\delta K_{2} d s\right) r m s .
$$

The dSext is calculated for each kind of magnets and listed in Table VIII. The sextupole imperfections down-fed from the octupoles are

$$
\text { dSext }=\operatorname{Nerr} 3\left(\delta K_{3} d s\right) \text { rms Xo }(2 / \pi) \text {. }
$$

The results of calculations are listed also in Table VIII. The imperfection by the first kind of down-feeding was much weaker than that by the magnet imperfections because there was less systematic octupole field. The estimated normal and skew sextupole imperfections were 3 or 4 times smaller than the observed values.

Table VII Observed strength of sextupole imperfections.

\begin{tabular}{|c|c|c|}
\hline resonance & normal & skew \\
\hline $3 Q x=14$ & $40 \pm 30$ & \\
\hline$Q x+2 Q y=14$ & $20 \pm 13$ & \\
\hline $2 \mathrm{Qx}+\mathrm{QY}=14$ & & $18 \pm 6$ \\
\hline $20 \mathrm{Q} x=9$ & $22 \pm 28$ & \\
\hline $2 Q Y=9$ & $40 \pm 18$ & \\
\hline$Q x+Q y=9$ & & $25 \pm 13$ \\
\hline average & $29 \pm 10$ & $20 \pm 6$ \\
\hline
\end{tabular}
The unit is $\mathrm{mrad} / \mathrm{m}^{2}$. 
Table VIII Estimation of the strengths of the normal and the skew sextupole harmonic imperfections.

\begin{tabular}{|c|c|c|c|c|c|}
\hline magnet Nerr & main field & \multicolumn{2}{|c|}{$\begin{array}{l}\text { normal sextupole } \\
\mathrm{B} 2 / \mathrm{ma} \text { in dSext } \\
\text { rms }\left(\mathrm{mrad} / \mathrm{m}^{2}\right)\end{array}$} & \multicolumn{2}{|c|}{$\begin{array}{l}\text { skew sextupole } \\
\text { A2/main dSext } \\
\text { rms (mrad } / \mathrm{m}^{2}\end{array}$} \\
\hline \multicolumn{6}{|c|}{ magnet imperfections } \\
\hline $\mathrm{B} \quad 36$ & $2 \pi / 36$ & $X 9 . E-3$ & 9.4 & $X 4 \cdot E-3$ & 4.2 \\
\hline $\mathrm{QF}$ & 0.270 & $X 1 \cdot E-3$ & 1.3 & $X 1 \cdot E-3$ & 1.3 \\
\hline $\mathrm{QD}$ & 0.276 & $X 1 \cdot E-3$ & 1.4 & $X 1 \cdot E-3$ & 1.4 \\
\hline SexF & 0.053 & $\times 3 \cdot E-3$ & 0.8 & $\times 2 \cdot E-3$ & 0.5 \\
\hline SexD & 0.003 & $\times 3 \cdot E-3$ & 0.1 & $\mathrm{X} 2 \cdot \mathrm{E}-3$ & 0.0 \\
\hline \multicolumn{2}{|l|}{ subtotal } & \multicolumn{3}{|c|}{9.6} & 4.6 \\
\hline & $\delta B 3\left(1 / \mathrm{m}^{3}\right)$ & $\mathrm{Xo}(\mathrm{mm})$ & dSext & Yo $(\mathrm{mm})$ & dSext \\
\hline \multicolumn{6}{|l|}{ down-feeding } \\
\hline$B \quad 36$ & $2 \pi / 36 \times 0.21$ & 1.1 & 0.92 & 0.3 & 0.25 \\
\hline $\mathrm{QF}$ & $0.270 \times 0.01$ & 0.6 & 0.02 & 0.3 & 0.01 \\
\hline QD $\quad 24$ & $0.276 \times 0.01$ & 1.0 & 0.04 & 0.2 & 0.01 \\
\hline SexF 24 & $0.053 \times 0.02$ & 0.6 & 0.01 & 0.3 & 0.00 \\
\hline SexD 24 & $0.003 \times 0.02$ & 1.0 & 0.00 & 0.2 & 0.00 \\
\hline \multicolumn{6}{|c|}{$8 \mathrm{~A} 3\left(1 / \mathrm{m}^{3}\right) \quad \mathrm{YO}(\mathrm{mm}) \mathrm{dSext} \quad \mathrm{XO}(\mathrm{mm}) \mathrm{dSext}$} \\
\hline \multicolumn{4}{|c|}{ 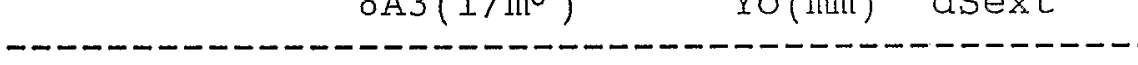 } & $\begin{array}{l}\lambda O(1 \mathrm{lll}) \\
------\end{array}$ & $\begin{array}{l}------ \\
\text { Usexu }\end{array}$ \\
\hline B 36 & $2 \pi / 36 \times 0.11$ & 0.3 & 0.13 & 1.1 & 0.48 \\
\hline $\mathrm{QF}$ & $0.270 \times 0.12$ & 0.3 & 0.15 & 0.6 & 0.30 \\
\hline $\mathrm{QD} \quad 24$ & $0.276 \times 0.11$ & 0.2 & 0.09 & 1.0 & 0.46 \\
\hline SexF 24 & $0.053 \times 0.02$ & 0.3 & 0.00 & 0.6 & 0.01 \\
\hline SexD 24 & $0.003 \times 0.02$ & 0.2 & 0.00 & 1.0 & 0.00 \\
\hline \multicolumn{2}{|l|}{ subtotal. } & \multicolumn{2}{|r|}{0.95} & & $0 . \overline{7}$ \\
\hline \multicolumn{2}{|l|}{ total } & \multicolumn{3}{|c|}{9.7} & 4.7 \\
\hline
\end{tabular}

IV-5 Octupole Imperfection

The strength of the octupole harmonic imperfection was measured at $B=1.7 \mathrm{kG}(\mathrm{B} p=2.36 \mathrm{Tm}), \mathrm{dB} / \mathrm{dt}=0 \mathrm{G} / \mathrm{ms}$, that was $2-3$ $\mathrm{rad} . / \mathrm{m}^{3}$ if we assume that the octupole imperfection of the $B$ term was much larger than that of off-set term.

The imperfection from the random variation of the magnets was estimated using the equation

$$
\left.\left.<\left(\Sigma \delta B 3 / \mathrm{B} \rho \mathrm{e}^{j 14 \theta}\right)^{2}\right\rangle=\mathrm{N}<(\delta \mathrm{B} 3 / \mathrm{B} \rho)^{2}\right\rangle \text {. }
$$


The estimated values from each imperfections are listed in Table IX. The total strength was only $0.17 \mathrm{rad} . / \mathrm{m}^{3}$, which was about 10 times smaller than the observed strength. There could be a strong remnant field imperfection or the other source of the imperfection. However we do not know what it was. The eddy-current correction windings, which had strong higher order multipoles, did not work because $\mathrm{dB} / \mathrm{dt}$ was 0 . The systematically distributed sextupoles could have produced only $6 n-t h$ harmonic octupole components. The quadrupole magnets have rather large skew octupole component but it is not normal and is systematic. Then none of them explains this large octupole imperfection. If there was unknown strong octupole field in the ring, it could be a reason why we observed a stronger sextupole imperfection than the expected.

Table IX Estimation of the octupole imperfections

\begin{tabular}{|c|c|c|c|c|c|}
\hline magnet & Nerr & ( $\delta \mathrm{B} 3) \mathrm{rms} / \mathrm{B} p$ & $\mathrm{~B} 4 / \mathrm{B} \rho$ & $\mathrm{X} 4 \delta \mathrm{xrms}$ & imperfection \\
\hline \multicolumn{6}{|c|}{ magnet imperfection } \\
\hline B & 36 & $2 \pi / 36 x$ & & & 0.15 \\
\hline$\overline{Q F}$ & 24 & $0.270 \times 0.01$ & & & 0.013 \\
\hline QD & 24 & $0.276 \times 0.01$ & & & 0.014 \\
\hline SexF & 24 & $0.053 \times 0.02$ & & & 0.005 \\
\hline SexD & 24 & $0.003 \times 0.02$ & & & 0.0003 \\
\hline \multicolumn{6}{|c|}{ down-feeding } \\
\hline $\mathrm{B}$ & 36 & & $2 \pi / 36 \times 9.8$ & $X 1.2 E-3$ & 0.074 \\
\hline tota & & & & & 0.17 \\
\hline
\end{tabular}




\section{$V \quad d B / d t$ TERM}

We observed considerably large dB/dt terms, which we did not expected. First we should check the system of the eddy-current field correction because we have experienced a failure of windings twice.

\section{V-1 Dipole imperfection}

The correction terms of harmonic C.O.D. was not parameterized but we know that the main dipole errors were B-term. Off-set term was much smaller than the $B$-term and $d B / d t$ term was negligibly smal1 [41]. E.Blesser observed a tiny dB/dt term which corresponded to a 3\% random error of the eddy-current correction system. This corresponded to $20 \%$ of the dipole $\mathrm{dB} / \mathrm{dt}$ strength of one correction winding [42].

The variation of resisters which were used to adjust the amplitudes of each correction winding was measured to be $2.7 \%$ [45] except three correction windings at F7, A4 and C5. A variation of the resistance was the same as the expected variation of the correction windings. This coincidence was maybe accidental because the resisters of each winding were adjusted to induce the same current $(I=15.8 \mathrm{~A}$ for $\mathrm{dB} / \mathrm{dt}=100 \mathrm{G} / \mathrm{ms}[37])$ in the correction coils.

The other possibility was an imperfection of dipole correction of the special beam duct at C5. As explained in Appendix $V$ the error would be as much as $20 \%$ of the total correction. This explains the amplitude of dipole $\mathrm{dB} / \mathrm{dt}$ term but the observed C.O.D. suggested unlocalized dipole error [42].

Let us check if the down-feeding of the quadrupole $\mathrm{dB} / \mathrm{dt}$ term could produce large dipole $\mathrm{dB} / \mathrm{dt}$ term. The strength of the quadrupole $\mathrm{dB} / \mathrm{dt}$ term was about 170 Gauss at dB/dt=100 G/ms ( Table III ). The $1 \mathrm{~mm}$ displace meant produced dipole field of $170 \mathrm{G} \times 2 \times$ $1 \mathrm{~mm} \div 2.42 \mathrm{~m}=0.14 \mathrm{G}$. This was more than 10 times smaller than the observed dipole imperfection.

\section{V-2 Normal quadrupole imperfection}

The AGS Booster has only one quadrupole eddy-current correction winding of the special vacuum chamber at C5. It could be misconnected or disconnected because it decoupled with dipole field unlikely to the sextupole correction windings. The phases of the observed imperfections are just at the C5 and the strength of them are close to the twice of the uncorrected eddy-current field. Then the misconnection of the winding with opposite polarity reproduces the observed imperfection as shown in Table $X$. The agreement was good enough to be suspicious about the connection of the winding. 
Table $X$ Observed normal quadrupole dB/dt term and the expected $\mathrm{dB} / \mathrm{dt}$ term when quadrupole correction winding at $\mathrm{C} 5$ is misconnected (Appendix V).

\begin{tabular}{|lrc|}
\hline correction string & observed & expected \\
\hline$\delta N(\cos 9 X) / \delta(d B / d t)$ & $5.5 \pm 2.8$ & 4.7 \\
$\delta N(\sin 9 X) / \delta(d B / d t)$ & $-1.5 \pm 1.3$ & -1.2 \\
$\delta N(\cos 9 Y) / \delta(d B / d t)$ & $3.36 \pm 0.11$ & 2.6 \\
$\delta N(\sin 9 Y) / \delta(d B / d t)$ & $-6.30 \pm 0.20$ & -3.6 \\
\hline
\end{tabular}

\section{V-3 Normal sextupole imperfections}

The strength of the observed sextupole dB/dt term is roughly the same as that produced by one correction winding as is calculated in Appendix V. But there could not be any disconnection of one winding because we did not observe strong dipole dB/dt term. In addition to this the connection at $F 7$ was fixed in 1992 , the connection at C5 (sextupole only) and A4 were checked during the run in 1993 [43], other connections than above three were checked after the run in 1993 [45].

One of 36 sextupole correction windings, the one at C5, is special and it cannot be perfect as explained in Appendix $V$ because the correction winding is displaced from the center of the vacuum chamber. First we will subtract this known dB/dt imperfection from the observed $\mathrm{dB} / \mathrm{dt}$ term. The results were listed in Table A-VI.

One possible source of the normal sextupole dB/dt term is a variation of eddy-current correction coils or vacuum chambers. A random variation of $10 \%$ rms would explain the observed imperfections. E.Blesser and R.Thern observed a residual dB/dt sextupole field when correction was applied. The strength was 0.01 $\mathrm{G} / \mathrm{cm}^{2}$ at $\mathrm{dI} / \mathrm{dt}=10 \mathrm{kA} / \mathrm{s}$, which corresponded to $15 \%$ of the correction [33]. However this measurement was not reliable because it. showed considerable non-linearity to the $\mathrm{dB} / \mathrm{dt}$, which did not show up in the other measurement by G.Danby and J.Jackson $[33,38]$. After all we have no evidence about the origin of the sextupole dB/dt term. Each group measured the field of only one magnet (because they did not have enough time) and it is impossible to measure all of the magnets with vacuum chambers from now.

\section{V-4 Other imperfections}

It was strange that we observed the considerable skew sextupole $\mathrm{dB} / \mathrm{dt}$ term although it was about 3 times smaller than the normal sextupole dB/dt term. We thought that the AGS Booster had no element which could produce such a large skew dB/dt term. We did not observe any skew quadrupole $\mathrm{dB} / \mathrm{dt}$ term neither but the error was considerably large. Actually we do not know much about the 
error source of the $\mathrm{dB} / \mathrm{dt}$ terms. We can only indicate the assistance of them. 


\section{OFF-SET TERM}

We observed a strong remnant field imperfection. There is no one location at which the phase fits the phases of all the observed imperfections. Then the remnant field might be distributed around the ring. Among three parameters: $\mathrm{Co}, \mathrm{Cb}$ and $\mathrm{Cbt}$ the fluctuation of Co was much larger than those of others [3].

One possible error source is a variation of remnant field of magnets. E.Blesser observed about $0.9 \mathrm{mT}$ field variation of the quadrupole magnets at low current [30], which explains the strength of the observed normal quadrupole off-set terms. However that variation could be only a measurement error.

The other possibility is a unusual magnetization. Some people thought that the nicrom heaters for the baking of vacuum chambers could be magnetized but that was not correct. The other one was a spot welding to $\mathrm{fix}$ a stainless steel tube of eddy-current correction winding on a vacuum chamber [46].

The strength of the remnant field components are listed in Table XI. At the full aperture ( $\mathrm{dX}=3 "=76.2 \mathrm{~mm}$ ) the field strength of each component was roughly the same except for the normal quadrupole component. This means that the remnant field changed transversely with the scale of the beam duct. At $d R=3$ " the integrated harmonic field strength was about $20 \mathrm{G} \mathrm{m}$. To estimate the strength of the error remnant field, we divide this by a half of the circumference of the ring. We also assumed that the number of random remnant field error source is roughly the same as the periodicity: 24 (this is also a number of locations with large $\beta x$ or $\beta y$, which are the weight functions of the strengths of resonances). The strength of the random remnant field error was estimated to be on the order of

$$
20 \mathrm{GmX} \sqrt{ } 24 / 100 \mathrm{~m}=1 \text { Gauss. }
$$

Which is rather weak and comparable to the Earth's field. The data about the strength of the systematic remnant field were listed in. Table A-III. The strength of the observed random remnant field was one order smaller than the systematic remnant field.

Table XII Strength of harmonic imperfection of the remnant field.

\begin{tabular}{|c|c|c|c|}
\hline field multipole & imperfection & B at & $\mathrm{dR}=3^{\prime \prime}$ \\
\hline normal quadrupole & $3 \times 10^{-3} \mathrm{~T}$ & 2 & $\mathrm{~m}$ \\
\hline skew quadrupole & $3 \times 10^{-2} \mathrm{~T}$ & $20 \mathrm{G}$ & $\mathrm{m}$ \\
\hline normal sextupole & $4 \times 10^{-1} \mathrm{~T} / \mathrm{m}$ & 20 & $\mathrm{~m}$ \\
\hline skew sextupole & $5 \times 10^{-1} \mathrm{~T} / \mathrm{m}$ & $30 \mathrm{G}$ & $\mathrm{m}$ \\
\hline ( normal octupole & $5 \times 10^{\circ} \mathrm{T} / \mathrm{m}^{2}$ & $20 \mathrm{G}$ & $\mathrm{m}$ \\
\hline
\end{tabular}




\section{DISCUSSIONS}

VII-1 Estimation of imperfections

Several estimations of the imperfection of the AGS Booster are listed in Table XIII. Mainly $B$ terms of the AGS Booster were estimated before the construction to design the maximum power of the correction elements because in usual case the maximum power is required to cancel the $B$ term at the top energy.

A displacements of magnets was much larger than any preestimation. We believed that this displacement was the main error source of the dipole and the normal quadrupole $B$ term. It was accidental that the observed C.O.D. amplitude was in the expected range [42] because the magnet production errors had been overestimated and the misalignment had been much underestimated. The accuracy of the alignment of the AGS Booster was rather poor in 1993 that it will be re-aligned before the operation in 1994. The horizontal and the vertical rms misalignment was about $3 \mathrm{~mm}$ and $1 \mathrm{~mm}$, respectively in 1993. They were about 10 and 3 times worse than the expected. The misalignment was also the reason why we observed the larger quadrupole stop-band than the expected.

The estimation of the systematic sextupole filed had been wrong. The edge sextupole field of the dipole magnet is the largest component of the systematic sextupole field but we did not have considered this field. On the other hand the eddy-current sextupoles had been considered to be the main sextupole component which were canceled by the correction windings.

The random quadrupole production error of the quadrupole magnets had been overestimated besides the random quadrupole error of the dipole magnets had not been considered, which contribution was larger than that of the quadrupole magnets.

The production error and the rotation of the chromaticity sextupole components are negligibly small. Then random sextupole field imperfections had been estimated as a fraction of the eddycurrent sextupoles. This estimation did not make sense because the eddy-current sextupoles were canceled. The main error source of the known sextupole imperfection of the real machine was a random sextupole component of the dipole magnet. However the observed strength of the imperfection was about 3 times larger than the present estimation. From a different point of view this estimation had been not bad because the $\mathrm{dB} / \mathrm{dt}$ term was larger than $\mathrm{B}$ term and off-set term. Although it did not mean that we had predicted the existence of the large normal sextupole $\mathrm{dB} / \mathrm{dt}$ term.

We had had less estimations about the skew sextupole imperfection. We could not even predict the assistance of the strong enough imperfection. This is why the skew sextupole correction was not introduced. 
Table XIII Estimations of the imperfections of the AGS Booster. The last column lists the estimation or measurement used in this report.

\begin{tabular}{|c|c|c|c|c|c|c|c|c|c|}
\hline reference & 24 & 47 & 48 & 49 & 50 & 44 & 20 & \multicolumn{2}{|c|}{22 present } \\
\hline \multicolumn{10}{|l|}{ DIPOLE } \\
\hline length of $B$ & 0.4 & 0.3 & & & & & & 0.3 & 0.3 \\
\hline $\operatorname{roli}$ of $B$ & 0.2 & 0.3 & & & 0.2 & & & 0.3 & $<5$ \\
\hline disp. of $Q$ & 0.1 & 0.3 & & & 0.1 & & & 0.3 & $\begin{array}{l}3(\mathrm{H}) \\
1(\mathrm{~V})\end{array}$ \\
\hline \multicolumn{10}{|l|}{ QUADRUPOLE } \\
\hline dX at eddy-s & & & & & & & 0.6 & & \\
\hline dx at chr-s & & & & & & & 0.3 & & 2 \\
\hline quad. of B & & & & & & & & & 0.9 \\
\hline Iength of $Q$ & & & 1 & & & & 0.6 & & 0.3 \\
\hline stop-band dQ & 0.7 & & & & & & & & 5 \\
\hline \multicolumn{10}{|l|}{ SKEW QUADRUPÕLE } \\
\hline \multicolumn{10}{|l|}{ sk-quad of $B$} \\
\hline roli of $Q$ & 0.5 & & & & 0.1 & 0.3 & 0.6 & & 0.5 \\
\hline dY at $\operatorname{chr}-\mathrm{S}$ & & & & & & 0.3 & 0.3 & & \\
\hline dY at eddy-S & & & & & & 0.3 & 0.6 & & \\
\hline \multicolumn{10}{|l|}{ NORMAI SEXTUPOLE } \\
\hline err of eddy-S* & $1 \%$ & & & $10 \%$ & & & 48 & & \\
\hline length of chr-s & 1 & & & 1 & & & 0.4 & & 3 \\
\hline \multicolumn{10}{|l|}{ SKEW SEXTUPOLE } \\
\hline roll of chr-s & 0.5 & & & & & & & & \\
\hline
\end{tabular}

* Eddy-current sextupole field was assumed to be $0.24 \mathrm{~T} / \mathrm{m}^{2}$

VII-2 Improvement of correction accuracy

The accuracy of the measured parameters were not enough and more accurate parameterization is required. The errors of the correction parameters listed in Table I were too large to cancel $2 Q y=14$ with enough accuracy [16]. The accuracy of third order resonance correction was not enough for the $\mathrm{Au}^{+33}$ operation $[51,52]$. Through our measurement we faced to some difficulties which should be removed to improve the accuracy.

The one was the variation of data points. Especially for the half-integer stop-band correction we found that the results on different dates were inconsistent with each other. Some other parameters such as chromaticity, C.O.D., dRset and any kind of bump orbit could have changed the results. When we attempt the same measurement again, these parameters shall be under control. Especially the required precision for the C.O.D. is high and the chromaticity should be set to an appropriate value.

The acceptable stop-band width, in other words the required 
accuracy for the correction, of half-integer resonances may be roughly 20E-5. Even with such a small stop-band we observed a large beam loss at the low intensity operation [16]. This accuracy is not enough but may be acceptable when the space-charge tune-spread is very large. This width is comparable to the contribution of downfeeding from the normal octupole imperfection. When chromaticity sextupoles were excited to cancel the chromaticities to 0s the required accuracy of C.O.D. control is about $0.05 \mathrm{~mm}$ (rms). And even when we turn off the chromaticity sextupoles the required accuracy of C.O.D. control is about $0.1 \mathrm{~mm}$. That accuracy is still not easy to be realized [42]. If we excite the sextupole magnets to cancel the edge sextupole field of the bending dipoles the tolerance of the C.O.D. control will become larger. In that case the chromaticities will come closer to the natural chromaticities from the bare machine chromaticities. A change of the chromaticity correction strings will be better. We have less reason to require higher periodicity of chromaticity correction sextupoles (now the periodicity is 24) because the Booster has strong systematic sextupole of bending dipoles with the periodicity 6 .

The correction parameters were searched with low intensity near the target resonance. But the programmed tune with high intensity is much higher than the resonance. When the quadrupole error was produced by a variations of quadrupole magnets, the required correction depends on the quadrupole strength. For example, the correction parameters of $2 Q Y=9$ are measured at $Q Y=4.5$ although the Booster is operated at $Q Y=4.96$. The correction should be about $10 \%$ stronger because the strength of quadrupole field is roughly proportional to the tune. The stop-band width of 2Qy=9 by the variation of the quadrupole magnets were about $1 . \mathrm{E}-3$ (Table IV). Then the stop-band width should be different by about $1 . E-4$ near the injection. The change is about a half of the tolerance. This gives the limit of correction accuracy of the stop-band $2 Q Y=9$ because we cannot measure the quadrupole imperfection of quadrupole magnets separately.

The dependence of 9 th harmonic C.O.D. on the tune also changes the quadrupole correction. The 9 th harmonic component of the C.O.D. will be proportional to $Q Y^{2} /\left(9^{2}-Q Y^{2}\right)$. Then the change of the C.O.D. amplitude amounts to $30 \%$. That effect should be minimized by reducing the systematic sextupole field or re-adjusting the C.O.D. We must be careful about the procedure of tuning parameters at the injection.

A ripple of the magnetic field and the dB/dt term will produce a fluctuation of the correction. Let us estimate the fluctuation of $2 Q y=9$ correction. When ripple frequency is $360 \mathrm{~Hz}$ and its amplitudes is $2 \mathrm{kG} \times 10^{-4}$, the $\mathrm{dB} / \mathrm{dt}$ will be

$$
\mathrm{dB} / \mathrm{dt}=2 \pi \times 360 \times 2 \times 10^{3} \times 10^{-4}=0.452 \mathrm{G} / \mathrm{ms}
$$

The amplitude of $\mathrm{dB} / \mathrm{dt}$ term was measured to be about 7.1 (Table I). Then the fluctuation of the stop-band width by the ripple will be 
$\pm 3.2 \mathrm{E}-5$, which is several times smaller than the tolerance. However if the ripple is larger than the assumed value, that effect would be considerable. To avoid such a disturbance we should manage to eliminate the source of quadrupole $\mathrm{dB} / \mathrm{dt}$ term, and we think it may be possible.

One of the other difficulty was a lack of power of the skew sextupole correction strings. We could not realize the optimum correction of $3 Q y=14$ [11] neither of the slope of $Q x+Q y=9$ [10]. The coupling of skew sextupole correction field with vertical dipole was also a problem. Those will be improved in the next year [39].

The third difficulty was that we did not have enough data points for various $B$ and $\mathrm{dB} / \mathrm{dt}$ to cross check the data. Because that kind of measurement requires long study time. We are still not sure whether the number of fitting parameters were enough or not.

At the high momentum or for a weak resonance, we failed to produce any beam loss [11]. Although the effect of resonances were weak at these points, the data at these points were still necessary for the parameterization. A very slow crossing helps the accuracy but we could not do much slower when dB/dt was not zero. The emittance measurement by the IPM would have provided more information. But it would have taken much more time.

Anyway the parameters listed in Table I is going to be revised with higher accuracy and with more resonances, such as 13 th sextupole resonances and octupole resonances. These data will give us more information about the imperfections.

VII-3 Other comments

The second kind of down-feeding is a very good tool to estimate the strength of higher order imperfections. There is a plan to introduce a correction system of octupole imperfection to grade-up the stop-band correction of the Booster [39]. We can estimate the required strength of correction easily before the installation.

There are so much parameters if we want to cancel the resonances in the region $4<\mathrm{Qx}, \mathrm{QY}<5$. Number of parameters will be more than 90. We must find more efficient way to handle such a large number of parameters.

\section{ACKNOWLEDGEMENT}

Many people contributed to the specification, design, construction, operation and studies of the Booster. The authors were only involved in the measurement and final analysis. We greatfully thank these AGS members for their information, discussion and support. 


\section{REFERENCES}

[1] Y.Shoji and C.Gardner, 'Half Integer Stop Band Width of the Booster', AGS SR-282, 1993.

[2] Y.Shoji and C.Gardner, '14th Normal Sextupole Correction', AGS SR-286, 1993.

[3] Y.Shoji and C.Gardner, '2QX $=9$ Correction Data Before May 7', AGS SR-287, 1993

[4] Y.Shoji and C.Gardner, '2QY $=9$ Correction Data Before May 7', AGS SR-288, 1993

[5] Y.Shoji and C.Gardner, 'Integer Coupling $(Q x+Q y=9)$ Correction Data', AGS SR-289, 1993

[6] Y.Shoji and C.Gardner, 'Tune Space Survey at Low Intensity (1)', AGS SR-290, 1993

[7] Y.Shoji, C.Gardner and C. Whalen, 'Observed Loss by 4th Resonances', AGS SR-291, 1993

[8] Y.Shoji and C.Gardner, 'Harmonic Component of C.O.D.', AGS SR-292, 1993

[9] Y.Shoji and C.Gardner, ' 9 th Normal Sextupole Correction Test', AGS SR-293, 1993

[10] Y.Shoji and C.Gardner, '9th Skew Sextupole Correction Test', AGS SR-294, 1993

[11] Y.Shoji and C.Gardner, 'Skew Sextupole Correction for $3 Q Y=14$ and $2 Q x+Q Y=14$ ', AGS SR295,1993

[12] Y.Shoji and C.Gardner, 'Tune Space Survey at Low Intensity', AGS SR-296, 1993

[13] Y.Shoji and C.Gardner, 'Intensity Dependence of resonances', AGS SR-297, 1993

[14] Y.Shoji and C.J.Gardner, 'Quadrupole and Sextupole correction Parameters for $20 y=9$ ', AGS SR-298, 1993

[15] Y.Shoji and C.Gardner, 'The Effect of the Skew Sextupole Correction', AGS SR-299, 1993

[16] Y.Shoji and C.Gardner, 'Structure Resonances', AGS SR-300, 1993

[17] Y.Shoji. and C.Gardner, 'Down Feed Matrix', AGS SR-305, 1993.

[18] Y.Shoji and C.Gardner, 'C.O.D. Analysis', AGS SR-307, 1993.

[19] Y.Shoji and C.Gardner, 'Down feed Effect Observed at the AGS Booster and Octupole Imperfection', AGS SR-308, 1993.

[20] S.Tepikian 'The Resonance Correction Scheme for the AGS Booster' ', Booster TN-149, Sept.27, 1989.

[21] S.Y.Lee and S.Tepikian, 'The Resonance Correction Scheme for the AGS Booster', Booster $\mathrm{TN}-150,10 / 25 / 89$

[22] J.Milutinovic, A.G.Ruggiero, S.Tepikian, and W.T.Weng ' AGS-Booster Orbit and Resonance Correction ', IEEE PAC'89, 
p. 1367

[23] C.Gardner,

'Booster Stopband Corrections', Booster TN-217, Jan.6, 1993.

[24] Y.Y.Lee,

'Requirement of the AGS Booster Correction elements', Booster $\mathrm{TN}-9,2 / 12 / 86$

[25] C.J.Gardner,

'Observation and Correction of Resonance Stopbands in the AGS Booster', AGS SR-273, Oct.12,1992.

[26] R.K.Reece, I.A.Ahrens, E.J.Blesser, J.M.Brennan, C.J.Gardner, J.W.Glenn, T.Roser, Y.Shoji, W.van Asselt, and W.T.Weng,

'On the High Intensity Aspects of AGS Booster Proton Operation', IEEE PAC'93.

[27] C.Gardner, Y.Shoji, L.Ahrens, J.W.Glenn, Y.Y.Lee, T.Roser, A.Soukas, W.van Asselt, and W.T.Weng,

'Observation and Correction of Resonance Stopbands in the AGS Booster', IEEE PAC'93.

[28] R.Thern

'Booster Dipole Production Measurements', Booster TN-190, March 13, 1991.

[39] E.Blesser

'Booster Long Quadrupole Production Measurements', Booster TN176, Sept.13, 1990.

[30] E.Blesser

'Booster Short Quadrupole Production Measurements I', Booster TN-174, Sept.12, 1990.

[31] E.BIesser

'Booster Sextupole Production Measurements I', Booster TN182, March 13, 1991.

[32] G.T.Danby and J.W.Jackson

'Static and Dynamic Magnetic End Effects and Correction Magnets for the AGS Booster', IEEE PAC 1989, p.381.

[33] E.Blesser and R.Thern

'Analysis of Magnetic Field Measurement Results foe the AGS Booster Magnets', IEEE PAC 1991, p.45.

[34] K. Zeno,

AGS Booster Commissioning 1993, Book VII, p.46.

[35] W.van Asselt, private communication, 1993.

[36] A.Iuccio and M.Blaskiewicz,

'AGS Booster Parameters (MAD output)', Booster TN-196, JulY $23,1991$.

[37] G.T.Danby and J.W.Jackson,

'Description of new vacuum chamber correction concept', IEEE PAC 1989, p.384.

[38] G.T.Danby and J.W.Jackson,

'Vacuum Chamber Eddy Current Self-Correction for the AGS Booster Accelerator', HEACC 1989, p.[279]/33.

[39] G.T.Danby, J.W.Jackson and C.Spataro

'Eddy Current Control in the AGS Rapid Cycling Booster Accelerator Magnets', presented at 13th International Conference on Magnet Technology, Victoria, B.C., Canada, Sept. $20-24,1993$.

[40] K.Brown, G.Murdock, S.Tanaka, C.Whalen and K.Zeno, 'Orbit Harmonics VS Tune in the Booster', AGS SR-280, April 


\section{$19,1993$.}

[41] L.Ahrens and T.Roser, AGS Booster Commissioning 1993, Book VI, p.52, AGS Booster Commissioning 1993, Book VII, p.58, and AGS Booster Commissioning 1993, Book IX, p.36.

[42] E.Blesser 'Results from Commissioning the AGS Booster Orbit System',

\section{IEEE PAC'93}

[43] E.Blesser and C.Whalen, 'Measurement of $\mathrm{C5}$ and A4 Eddy Current Correctors', AGS SR281, April 21, 1993.

[44] S.Tepikian

' Skew Quadrupole Corrections ', TN-132, Oct.10, 1988.

[45] E.Blesser, private communication, 1993

[46] H.C.Hseuh, private communication, 1993.

[47] J.Milutinovic and A.G.Ruggiero

' Closed Orbit Analysis for the AGS Booster', Booster TN-107, Feb.1, 1988 .

[48] J.Milutinovic and A.G.Ruggiero, 'Effects of Quadrupole Gradient Errors in the AGS booster', Booster TN-112, 2/23/88

[49] S.Tepikian

' Random Sextupole Correction', Booster TN-125, Aug.5, 1988.

[50] A.Ruggiero, AGS Booster design manual, p.2-52, revised in Oct., 1988 .

[51] K.Zeno, Booster Book XII, HIP Running/Setup, p.7.

[52] Y.Shoji, Booster Book XI, HIP Running/Setup, p.93.

[53] A. Luccio, 'Algorithm and Charts to Calculate and Modify Tunes and Chromaticity in the AGS Booster, Proton Case', Booster TN-179, Oct.17, 1990 .

[54] W. van Asselt, private communication, 1993.

[55] W. van Asselt and I. Ahrens, 'Chromaticity measurements at the AGS Booster', AGS SR264 , Oct. 2,1991 .

[56] W. Van Asselt and I. Ahrens, 'Chromaticity measurements in the AGS Booster', AGS SR-269, Sept.9, 1992.

[57] Y.Y. Lee, private communication, 1993. 
Appendix I Magnet Production Errors

Here we reprint the results of field measurement of the magnets for a convenience of readers. The original reports are listed as references. Any known misprint in the original is revised. Some additional analysis by us is added. The units are changed from the original for our convenience. The definition of multipole components of magnetic fields are given as equations (II4).

Table A-I Harmonic component of integrated field of the dipole magnets by $R$.Thern [28]. The special magnet used at C5 was not included. The misprinted units in reference is corrected. The current region used for the proton injection is $2600 \mathrm{~A}$. The average is the systematic imperfection and the rms is the root mean square of the random imperfection.

\begin{tabular}{|c|c|c|c|c|c|}
\hline \multirow{2}{*}{$\begin{array}{l}\text { harmonics } \\
\text { current }\end{array}$} & \multicolumn{2}{|c|}{ average } & \multicolumn{2}{|c|}{$\mathrm{rms}$} & \multirow[t]{2}{*}{ unit } \\
\hline & $2600 \mathrm{~A}$ & $5000 \mathrm{~A}$ & $2600 A$ & $5000 \mathrm{~A}$ & \\
\hline $\mathrm{BO}$ & & & 15. & 30 & $E-5$ \\
\hline $\mathrm{A} 0 / \mathrm{BO}$ & & & 4.9 & 5.4 & $E-5$ \\
\hline $\mathrm{B} 1 / \mathrm{B} 0$ & & & 9.1 & 8.6 & $E-4 / m$ \\
\hline $\mathrm{A} 1 / \mathrm{B} 0$ & 2.4 & 6.0 & 4.0 & 4.8 & $E-4 / m$ \\
\hline $\mathrm{B} 2 / \mathrm{B} 0$ & -24 & -64 & 0.9 & 0.8 & $\mathrm{E}-2 / \mathrm{m}^{2}$ \\
\hline $\mathrm{A} 2 / \mathrm{BO}$ & -0.075 & -0.014 & 0.4 & 0.6 & $\mathrm{E}-2 / \mathrm{m}^{2}$ \\
\hline $\mathrm{B} 3 / \mathrm{B} 0$ & 2.1 & 5.3 & 1.4 & 1.3 & $\mathrm{E}-1 / \mathrm{m}^{3}$ \\
\hline $\mathrm{A} 3 / \mathrm{BO}$ & 1.1 & 1.5 & 0.8 & 0.9 & $E-1 / m^{3}$ \\
\hline $\mathrm{B} 4 / \mathrm{BO}$ & -9.8 & -87 & 1.1 & 1.1 & $\mathrm{E}+0 / \mathrm{m}^{4}$ \\
\hline $\mathrm{A} 4 / \mathrm{B} 0$ & 0.14 & 0.47 & 0.9 & 0.8 & $\mathrm{E}+0 / \mathrm{m}^{4}$ \\
\hline $\mathrm{B} 5 / \mathrm{BO}$ & 5.5 & 12 & 5.9 & 5.4 & $\mathrm{E}+1 / \mathrm{m}^{5}$ \\
\hline $\mathrm{A} 5 / \mathrm{BO}$ & -2.2 & -0.53 & 2.1 & 1.9 & $\mathrm{E}+1 / \mathrm{m}^{5}$ \\
\hline $\mathrm{B} 6 / \mathrm{B} 0$ & -0.24 & -91 & 5.6 & 4.9 & $\mathrm{E}+2 / \mathrm{m}^{6}$ \\
\hline $\mathrm{A} 6 / \mathrm{B} 0$ & 0.87 & -0.61 & 3.2 & 3.2 & $\mathrm{E}+2 / \mathrm{m}^{6}$ \\
\hline
\end{tabular}


Table A-II Harmonic component of the integrated field imperfection reported by E.Blesser of long quadrupole magnets [29] and short quadrupole magnets [30] at the current 2600A. The field of 4 long quadrupoles and 14 short quadrupoles were measured. These data were revised in the conference report [33] without details. The $B 0 / B 1, A 0 / A 1$ and $A 1 / B 1$ were considered to be a horizontal displacement, a vertical displacement and a rotation of magnet, respectively. The result of the measurement of the prototype quadrupole magnet reported by G.T. Danby and J.W.Jackson was listed in the column of the reference [32]. The average (systematic) error of $\mathrm{B} 5 / \mathrm{BI}$ are allowed by mechanical symmetry.

\begin{tabular}{|c|c|c|c|c|c|c|c|}
\hline \multirow[t]{2}{*}{ harmonics } & \multicolumn{2}{|c|}{ average } & \multicolumn{2}{|c|}{ rms } & \multirow{2}{*}{$\begin{array}{l}\text { ref. } \\
\text { [33] }\end{array}$} & \multirow{2}{*}{$\begin{array}{l}\text { ref. } \\
{[32]}\end{array}$} & \multirow[t]{2}{*}{ unit } \\
\hline & long & short & long & short & & & \\
\hline $\mathrm{B} 0 / \mathrm{B} 1$ & 34.4 & -17.6 & 0.7 & 0.8 & & -0.13 & $\mathrm{E}-5 \mathrm{~m}$ \\
\hline $\mathrm{AO} / \mathrm{BI}$ & $-41 \cdot 9$ & 6.0 & 1.2 & 1.1 & & -1.1 & $\mathrm{E}-5 \mathrm{~m}$ \\
\hline $\mathrm{B} 1 / \mathrm{B} 1$ & & & 1.8 & 1.6 & 2.6 & & $E-4$ \\
\hline $\mathrm{A} I / \mathrm{B} I$ & -7.09 & -0.63 & 0.7 & 0.5 & & & $E-4$ \\
\hline $\mathrm{B} 2 / \mathrm{B} 1$ & -3.25 & -3.18 & 2.5 & 2.2 & 10 & -32 & $E-4$ \\
\hline $\mathrm{A} 2 / \mathrm{B} 1$ & -22.3 & 6.22 & 1.7 & 2.3 & & -164 & $E-4 / \mathrm{m}$ \\
\hline $\mathrm{B} 3 / \mathrm{B} 1$ & 5.59 & 1.79 & 1.3 & 3.4 & 10 & -13 & $\mathrm{E}-3 / \mathrm{m}^{2}$ \\
\hline $\mathrm{A} 3 / \mathrm{B} 1$ & -106 & -116 & 3.5 & 3.0 & & -92 & $\mathrm{E}-3 / \mathrm{m}^{2}$ \\
\hline $\mathrm{B} 4 / \mathrm{B} 1$ & -7.7 & 27.7 & 6.9 & 8.5 & 10 & -14 & $\mathrm{E}-2 / \mathrm{m}^{3}$ \\
\hline $\mathrm{A} 4 / \mathrm{B} 1$ & -12.2 & 34.4 & 7.7 & 9.8 & & 29 & $\mathrm{E}-2 / \mathrm{m}^{3}$ \\
\hline $\mathrm{B} 5 / \mathrm{B} 1$ & 10.9 & 8.60 & 2.3 & 1.8 & 4 & 0.6 & $\mathrm{E}+0 / \mathrm{m}^{4}$ \\
\hline $\mathrm{A} 5 / \mathrm{B} 1$ & -6.01 & -8.53 & 2.3 & 2.7 & & 43 & $\mathrm{E}+0 / \mathrm{m}^{4}$ \\
\hline
\end{tabular}

Table A-III Residual (remnant) fields of the quadrupole magnets $[29,30]$. The rms was deduced by Y.Shoji from Figure 3 of the reference [30]. The dB/dt column lists the change of the remnant field by the difference of $\mathrm{dB} / \mathrm{dt}(11 \mathrm{~T} / \mathrm{s})$.

\begin{tabular}{|c|c|c|c|c|c|c|}
\hline magnet & field & average & rms & $d B / d t$ & unit & reference \\
\hline \multirow[t]{3}{*}{ Dipole } & BO & 19 & & & Gauss m & [28] \\
\hline & $\mathrm{BO}$ & 15 & & & Gauss m & [39] \\
\hline & $\mathrm{BO}$ & $13-22$ & 9 & & Gauss m & {$[32]$} \\
\hline \multirow[t]{4}{*}{ Quad. } & $\mathrm{BO}$ & 0.5 & & & Gauss $m$ & {$[29,30]$} \\
\hline & $\mathrm{AO}$ & 0.01 & & & Gauss $m$ & {$[29,30]$} \\
\hline & B1 & 25 & & 1 & Gauss & {$[29,30]$} \\
\hline & $\mathrm{A} 1$ & 0.7 & & & Gauss & {$[29,30]$} \\
\hline
\end{tabular}


Table A-IV Harmonic component of integrated field of the sextupole magnet reported by E.Blesser [31] and [33] by E.Blesser and R.Thern [33].

\begin{tabular}{|c|c|c|c|c|c|}
\hline $\begin{array}{l}\text { harmonics } \\
\text { reference }\end{array}$ & $\begin{array}{c}\text { average } \\
31\end{array}$ & $\begin{array}{l}\text { rms } \\
31\end{array}$ & 33 & unit & \\
\hline $\mathrm{B} 0 / \mathrm{B} 2$ & & 18 & & $E-6$ & $\mathrm{~m}^{2}$ \\
\hline $\mathrm{A} 0 / \mathrm{B} 2$ & & 2.4 & & $E-6$ & $\mathrm{~m}^{2}$ \\
\hline $\mathrm{B} 1 / \mathrm{B} 2$ & & 2.4 & & $E-4$ & $\mathrm{~m}$ \\
\hline $\mathrm{A} 1 / \mathrm{B} 2$ & & 2.8 & & $E-4$ & $\mathrm{~m}$ \\
\hline $\mathrm{B} 2 / \mathrm{B} 2$ & & 3.4 & 3 & $E-3$ & \\
\hline $\mathrm{A} 2 / \mathrm{B} 2$ & -24 & 2.2 & & $E-3$ & \\
\hline $\mathrm{B} 3 / \mathrm{B} 2$ & 1.3 & 2.0 & 2 & $E-2$ & $/ \mathrm{m}$ \\
\hline $\mathrm{A} 3 / \mathrm{B} 2$ & 0.28 & 2.5 & & $\mathrm{E}-2$ & $/ \mathrm{m}$ \\
\hline $\mathrm{B} 4 / \mathrm{B} 2$ & -7.2 & 7.5 & 7 & $\mathrm{E}-1$ & $/ \mathrm{m}^{2}$ \\
\hline $\mathrm{A} 4 / \mathrm{B} 2$ & 10 & 8.4 & & $E-1$ & $/ \mathrm{m}^{2}$ \\
\hline $\mathrm{B} 5 / \mathrm{B} 2$ & 1.9 & 2.3 & 2 & $E+1$ & $/ \mathrm{m}^{3}$ \\
\hline A5/B2 & -1.1 & 1.9 & & $E+1$ & $/ \mathrm{m}^{3}$ \\
\hline
\end{tabular}


Appendix II Calculation memo about the correction parameter unit

The strength of integrated correction field when $N(x \times x x)=1$ is summarized. The values were calculated using definitions reported by C.Gardner [23]. A normalization constant of the skew quadrupole correction was changed after the Booster Tech. Note was written by $C$. Gardner from $10^{5} / 4 \pi$ to $10^{5} / 8 \pi$. The matrix for $Q x-Q Y=0$ was also changed to save power of the correction string but the definition of $N$ (COsOXY) did not change. The connection of magnets for 9 th normal sextupole strings; SH4 and SV4 and skew sextupoles and strength of skew sextupole correction field were summarized in the reference [17] according to informal reports by A.Soukas, J.Jackson, G.Danby et al. The polarities of correction strings were checked on October 28 by $\mathrm{C}$. Whalen and authors.

(1) $2 Q \mathrm{QX}=9,2 Q \mathrm{Q}=9, \mathrm{QX}-Q \mathrm{QY}=0, Q \mathrm{QX}+Q \mathrm{Q}=9, \quad 3 Q \mathrm{QX}=14$ and $Q \mathrm{QX}+2 \mathrm{QY}=14$.

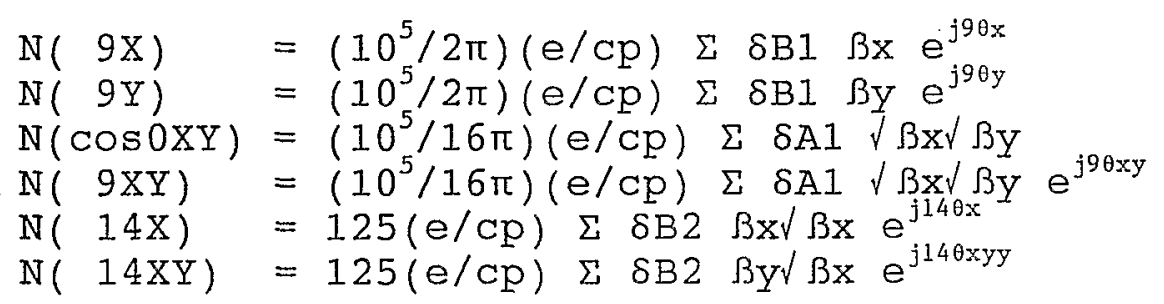

$$
(\mathrm{CP} / \mathrm{e})=3.335641(\mathrm{Tm} /(\mathrm{GeV} / \mathrm{C}))
$$

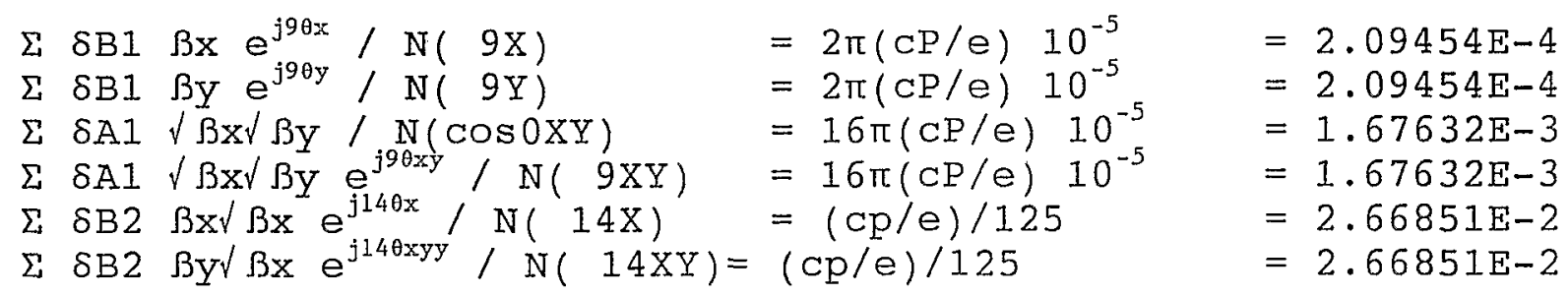

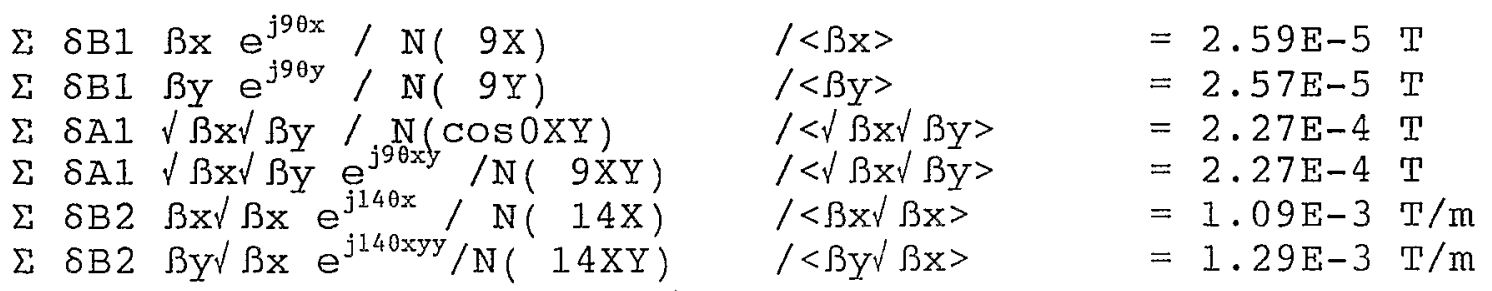

polarity -- applied correction with positive parameter $\begin{array}{lll}\text { quadrupole } & -- & \mathrm{d} \phi \mathrm{x} / \mathrm{dx}<0 \\ \text { skew quadrupole } & -- & \mathrm{d} \phi \mathrm{y} / \mathrm{dx}>0 \\ \text { sextupole } & --- & \mathrm{d}^{2} \phi \mathrm{dx} / \mathrm{dx}^{2}>0\end{array}$

phase $\quad-\theta=0$ at the beginning of the super period $A$

(2) Skew sextupoles; $Q Y+2 Q X=14$ and $3 Q y=14$

¿ SAI ßx/ By $e^{j 140 x} / S V 3=6.58 \mathrm{E}-2$ 


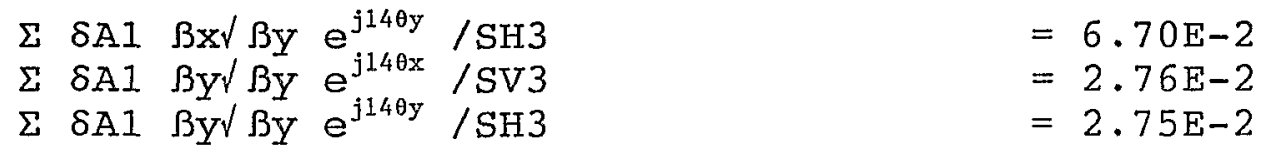

$$
\begin{aligned}
& \mathrm{N}=2 \\
& \delta A 1 / \mathrm{SH} 3=\delta A 1 / \mathrm{SV} 3=13.3 \mathrm{E}-4 \mathrm{~T} / \mathrm{m} / \mathrm{A} \text {. } \\
& B x \sqrt{ } B y=24.73 \mathrm{~m} \quad B y / B y=10.38 \mathrm{~m} \\
& =25.19 \mathrm{~m} \quad=10.35 \mathrm{~m}
\end{aligned}
$$
$\Sigma \delta A 1 \beta x / B y e^{j 14 \theta x}$
$\Sigma \delta A 1 \beta x \sqrt{ }$ ßy $e^{j 14 \theta y} / \mathrm{SH} 3$
$/<\beta x / \beta y>$
$=3.20 \mathrm{E}-3 \mathrm{~T}$
$\Sigma$ SA1 ßyل BY $e^{j 14 \theta x} / S V 3$
$/<\beta x / \beta Y>$
$=3.26 \mathrm{E}-3 \mathrm{~T}$
$\Sigma$ SAI ßYV BY $e^{j 14 \theta y} /$ SH3
$/<\beta y \mid$ By $>$
$=1.10 \mathrm{E}-3 \mathrm{~T}$
$/<\beta y /$ By $>$
$=1.11 \mathrm{E}-3 \mathrm{~T}$

(SV3)

(SH3)

polarity -- applied correction with positive parameter skew sextupole

$\ldots \mathrm{d}^{2} \phi \mathrm{y} / \mathrm{dx} \mathrm{x}^{2}>0$

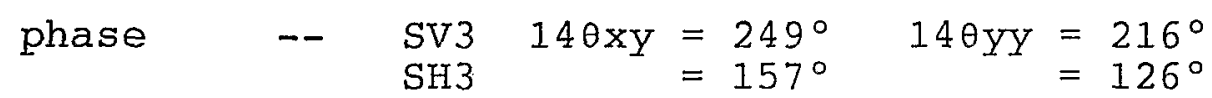

(3) slopes of $2 Q x=9,2 Q y=9, Q x+Q y=9$ and $3 Q x=14$

$\delta N(9 \mathrm{X}) /$ ddRset $=\left(10^{5} / 2 \pi\right)(\mathrm{e} / \mathrm{cp}) \Sigma 2 \delta B 2 \eta(\mathrm{dP} / \mathrm{P}) \quad B \mathrm{X} \mathrm{e}^{\mathrm{j} 9 \theta \mathrm{x}} \mathrm{j} / \mathrm{dRset}$ $\delta N(9 Y) / \delta d R s e t=\left(10^{5} / 2 \pi\right)(\mathrm{e} / \mathrm{cp}) \Sigma 2 \delta B 2 \eta(\mathrm{dP} / \mathrm{P})$ BY $e^{j 9 \theta y} / \mathrm{dRset}$ $\delta N(9 X Y) / \delta d R s e t$

$=\left(10^{5} / 16 \pi\right)(\mathrm{e} / \mathrm{cp}) \Sigma 2 \delta \mathrm{A} 2 \eta(\mathrm{dP} / \mathrm{P}) / \mathrm{Bx} / \mathrm{By} \mathrm{e}^{\mathrm{j} 9 \theta \mathrm{xy}} / \mathrm{dRs}$ et

$\delta \mathrm{N}(14 \mathrm{X}) / \delta \mathrm{dRset}=125(\mathrm{e} / \mathrm{cp}) \Sigma 3 \delta \mathrm{B} 3 \eta(\mathrm{dP} / \mathrm{P}) \quad \beta \mathrm{x} / \beta \mathrm{x} e^{j 14 \theta \mathrm{x}} / \mathrm{dRset}$

$\delta N(9 x) / \delta\left(\right.$ dRset $\left.^{2}\right)$

$=\left(10^{5} / 2 \pi\right)(\mathrm{e} / \mathrm{cp}) \Sigma 36 \mathrm{~B} 3[\eta(\mathrm{dP} / \mathrm{P})]^{2} B \mathrm{x} e^{j 9 \theta \mathrm{x}} /{\text { [dRset }]^{2}}^{2}$

$\delta \mathrm{N}(9 \mathrm{Y}) / \delta\left(\right.$ dRset $\left.^{2}\right)$

$=\left(10^{5} / 2 \pi\right)(\mathrm{e} / \mathrm{cp}) \Sigma 38 \mathrm{~B} 3[\eta(\mathrm{dP} / \mathrm{P})]^{2}$ By $\mathrm{e}^{\mathrm{j} 90 \mathrm{y}} /[\mathrm{dRset}]^{2}$

$(\mathrm{dP} / \mathrm{P}) /(\mathrm{dRset})=1 / 319$

$\Sigma \delta B 2 \eta \mathrm{Bx} \mathrm{e}^{\mathrm{j} 9 \mathrm{\theta x}} /[\delta \mathrm{N}(9 \mathrm{X}) / \delta \mathrm{dRset}]=319 \pi(\mathrm{CP} / \mathrm{e}) 10^{-5}=3.34 \mathrm{E}-2$

$\Sigma \delta B 2 \eta B Y e^{j 9 \theta y} /[\delta N(9 Y) / \delta d R s e t]=319 \pi(\mathrm{CP} / \mathrm{e}) 10^{-5}=3.34 \mathrm{E}-2$

$\Sigma$ SA2 $\eta \sqrt{ } B \mathrm{X} / \mathrm{BY} \mathrm{e}^{\mathrm{j} 90 \mathrm{xy}} /[\delta \mathrm{N}(9 \mathrm{XY}) / \delta \mathrm{dRset}]$

$=3198 \pi(\mathrm{CP} / \mathrm{e}) 10^{-5}=2.67 \mathrm{E}-1$

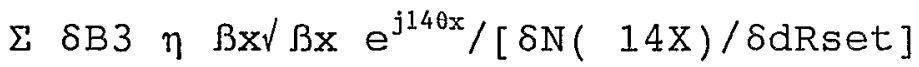

$=319(\mathrm{CP} / \mathrm{e}) / 375=2.84 \mathrm{E}-1$

$\Sigma \delta B 3 \eta^{2} \beta \mathrm{X} e^{j 9 \theta x} /\left[\delta \mathrm{N}(9 \mathrm{X}) / \delta\left(\right.\right.$ dRset $\left.\left.^{2}\right)\right]=319^{2} 2 \pi(\mathrm{CP} / \mathrm{e}) 10^{-5} / 3=7.11 \mathrm{E}+0$

$\Sigma \delta B 3 \eta^{2} B Y e^{j 9 \theta y} /\left[\delta N(9 Y) / \delta\left(\right.\right.$ dRset $\left.\left.^{2}\right)\right]$

$$
=319^{2} 2 \pi(\mathrm{CP} / \mathrm{e}) 10^{-5} / 3=7.11 \mathrm{E}+0
$$

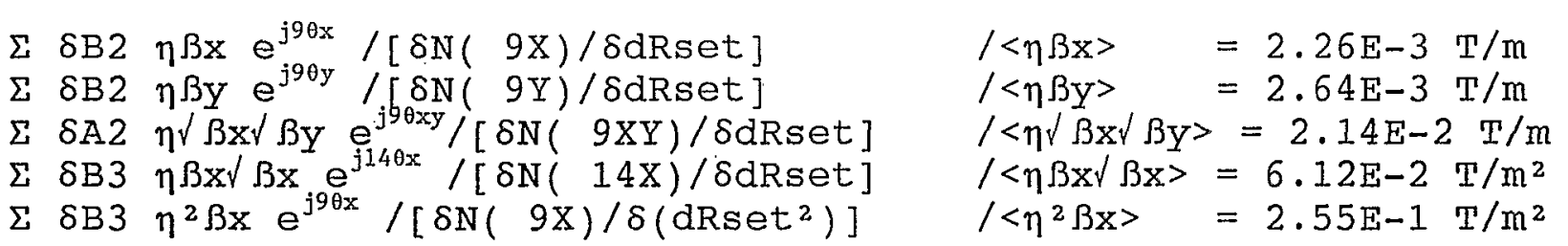


$\Sigma$ \&B3 $\eta^{2} \beta Y e^{j 9 \theta y} /\left[\delta N(9 Y) / \delta\left(\right.\right.$ dRset $\left.\left.^{2}\right)\right] \quad /\left\langle\eta^{2} \beta Y\right\rangle=3.48 \mathrm{E}-1 \mathrm{~T} / \mathrm{m}^{2}$

(4) 9 th normal sextupole for $2 Q \mathrm{Qx}=9$ and $2 \mathrm{QY}=9$.

$\Sigma \delta B 2 \eta B \mathrm{x} e^{j 9 \theta \mathrm{x}} / \mathrm{SV} 4=1.47 \mathrm{E}-1$

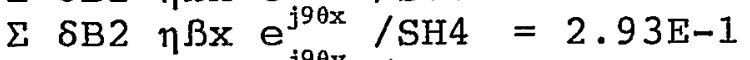

$\Sigma$ SB2 $\eta$ BY $e^{j 9 \theta y} / \mathrm{SV}^{j}=0.57 \mathrm{E}-1$

$\Sigma$ $\delta$ B2 $\eta$ BY $e^{j 9 \theta y} / \mathrm{SH} 4=1.13 \mathrm{E}-1$

$\Sigma$ 8B2 $\eta ß x e^{j 90 x} / S V 4 /<\eta \beta x>=0.99 E-2 \mathrm{~T} / \mathrm{m}$

$\Sigma$ SB2 $\eta \beta x e^{j 9 \theta x} / \mathrm{SH} 4 /<\eta \beta x>=1.98 \mathrm{E}-2 \mathrm{~T} / \mathrm{m}$

$\Sigma$ SB2 $\eta ß Y e^{j 9 \theta y} / \mathrm{SV} 4 /<\eta \beta Y>=0.46 \mathrm{E}-2 \mathrm{~T} / \mathrm{m}$

$\Sigma$ SB2 $\eta \beta Y e^{j 9 \theta y} / \mathrm{SH} 4 /<\eta \beta Y>=0.89 \mathrm{E}-2 \mathrm{~T} / \mathrm{m}$ 
Appendix III Expected correlation of two resonances.

Correction parameters of resonances produced by the same imperfection have correlation. Such pairs are $2 Q \mathrm{Q}=9$ and $2 Q \mathrm{Q}=9$ produced by the 9 th normal quadrupole imperfection, $30 x=14$ and $Q x+2 Q y=14$ produced by the 14 th sextupole imperfection and the slopes of $2 Q x=9$ and $2 Q y=9$ produced by the 9 th normal sextupole imperfection. Weight functions are different for the two of each pair but error sources are the same. A ratio of the weight function of the former of pairs to that of the latter is $\beta x / \beta y$. We will calculate the expected correlation when imperfection field are consist of two groups of random imperfection: one at $Q F$ ( $\delta F$; $\beta x / \beta y=3)$ and the other at $Q D(\delta D ; \beta y / \beta x=3)$. They are assumed to be random and independent to each other but their expected error amplitudes are the same. We use $A$ and $B$ as the strength of values of the former and the latter parameters.

$$
\begin{aligned}
& A=3 \delta F+\delta D \\
& B=\delta F+3 \delta D \\
& <\delta F^{2}>=\left\langle\delta D^{2}>\right. \\
& \langle\delta F \delta D>=0
\end{aligned}
$$

The correlation of the couple of resonances are:

$$
\frac{\left\langle(A-B)^{2}\right\rangle}{\sqrt{ }\left(\left\langle A^{2}\right\rangle\left\langle B^{2}\right\rangle\right)}=\frac{\left\langle(2(\delta F-\delta D))^{2}\right\rangle}{\left\langle(3 \delta F+\delta D)^{2}\right\rangle}=2 / 5 .
$$

We expect a meaningful correlation under the above assumption.

The correction phase between $A$ and $B$ is biased because the horizontal betatron phase advances more than the vertical betatron phase. The phase difference defined as

$$
\Delta \theta \equiv \mu x / Q X-\mu Y / Q Y
$$$$
(A-I I I-4)
$$

is shown in Table $A-V$. Here $\mu x$ and $\mu y$ were defined in section II. The $\Delta \theta$ varies from 0 to 4.7 degrees. Then the expected phase differences of the 9 th and 14th harmonic components are

$$
\begin{aligned}
& 9 \theta x-9 \theta y \approx 20 \text { degrees and } \\
& 14 \theta x-14 \theta y \approx 30 \text { degrees. }
\end{aligned}
$$

Figure $\mathrm{A}-1$ shows the expected correlation between correction parameters of $3 Q \mathrm{Qx}=14$ and $\mathrm{QX}+2 \mathrm{QY}=14$ under the above assumption. When a correction point of $3 \mathrm{Qx}=14$ is at the position indicated by the black spot the expected region for a correction point of $\mathrm{Qx}+2 \mathrm{QY}=14$ is shown as the circle. When the random errors located not at the quadrupoles the error circle becomes smaller according to the ratio of $\beta x$ and $\beta y$ while the mostly expected phase difference remains the same. 
Table A-V Calculated phase difference between horizontal and vertical betatron oscillation.

\begin{tabular}{|lr|}
\hline location & \multicolumn{1}{c|}{$\Delta \theta / 2 \pi$} \\
\hline Correction & $2.4 \mathrm{E}-3$ \\
SextD & $2.9 \mathrm{E}-3$ \\
QD & $6.0 \mathrm{E}-3$ \\
$\mathrm{~B}(1,5,7)$ in & $8.8 \mathrm{E}-3$ \\
B $(1,5,7)$ out & $11.2 \mathrm{E}-3$ \\
Correction & $9.6 \mathrm{E}-3$ \\
SextF & $9.0 \mathrm{E}-3$ \\
QF & $6.0 \mathrm{E}-3$ \\
B $(2,4,8)$ in & $3.3 \mathrm{E}-3$ \\
$\mathrm{~B}(2,4,8)$ out & $0.8 \mathrm{E}-3$ \\
\hline
\end{tabular}


Appendix IV Chromaticity of the AGS Booster

The chromaticity of the bare machine, which meant the machine with no correction, was not the same as the natural chromaticity because of the sextupole field of the bending dipoles. The chromaticity change from the (normalized) natural chromaticity can be calculated using the following equation.

$$
\delta \xi=(1 / 4 \pi) \Sigma 2(B 2 / B \rho) \eta B / Q \quad(A-I V-1)
$$

The sextupole fields locate at the both ends of dipoles [32]. Their strength at each of the edges; $B 2 / B \rho$ was measured by $R$. Thern (Appendix I) to be

$$
\mathrm{B} 2 / \mathrm{B} \rho=(2 \pi / 72) \times 0.24\left(/ \mathrm{m}^{2}\right)
$$

at the low field. The averages of $\eta ß$ at 72 dipole edges are

$$
\left\langle\eta \beta x > = 1 2 . 9 3 5 ( \mathrm { m } ^ { 2 } ) \text { and } \left\langle\eta \beta y>=11.597\left(\mathrm{~m}^{2}\right)\right.\right.
$$

when $Q x=4.633676$ and $Q y=4.583271$ [36]. Then the expected change of chromaticities are

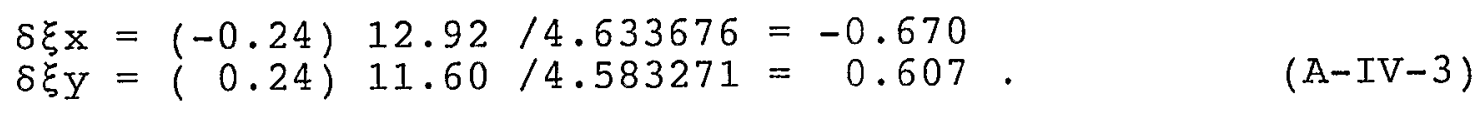

The natural chromaticities are calculated by $A$. Luccio to be -0.99 and -1.07 for horizontal and vertical, respectively [53]. Then the expected chromaticities are

$$
\begin{array}{ll}
\xi_{X}=-0.99-0.67 & =-1.66 \\
\xi_{Y}=-1.07+0.61 & =-0.46
\end{array}
$$$$
(\mathrm{A}-\mathrm{IV}-4)
$$

On the other hand the measured chromaticities by $W$. van Asselt were $\xi_{x}=-1.568$ and $\xi_{Y}=-0.623$ [54]. The agreement was not bad.

The harmonic sextupole imperfections were much smaller than the systematic sextupole. After all the strength of the edge sextupoles are

$$
\sum B 2 / B p=2 \pi \times 0.24=1.5\left(/ \mathrm{m}^{2}\right)
$$

and the strength of the harmonic component (in this case the 0th harmonic component) were roughly

$$
\sum \mathrm{B} 2 / \mathrm{B} \rho=0.03\left(/ \mathrm{m}^{2}\right) \text {. }
$$

The expected change of the normalized chromaticity due to the random sextupole imperfection is roughly 0.01 . No wonder the observed chromaticity did not depend on $B$ neither $d B / d t$ [54] although the harmonic imperfections had considerable $\mathrm{B}$ and $\mathrm{dB} / \mathrm{dt}$ dependence.

The typical chromaticity values during the machine studies 
about the stop-band were $\xi x=-0.5$ and $\xi y=-0.25$. (However the chromaticities during the high intensity operation were $\xi x=-0.5$ and $\xi_{Y}=-0.75$, maybe because the vertical chromaticity should be a larger negative value to suppress the single bunch instability). The average of the beta-function times the dispersion function at the horizontal chromaticity control sextupoles are

$$
\langle\eta \beta x\rangle=24.680 \text { and }\langle\eta \beta y\rangle=9.338 \text {, }
$$

and at the vertical chromaticity control sextupoles they are

$$
\langle\eta \beta x\rangle=6.019 \text { and }\langle\eta \beta y\rangle=16.133 \text {. }
$$

Then the chromaticity change by the sextupoles with strengths $\mathrm{k} 2 \mathrm{x}$ and $\mathrm{K} 2 \mathrm{y}$, horizontal and vertical respectively, are

$$
\begin{aligned}
& \left(\begin{array}{l}
\delta \xi \mathrm{x} \times 4.633676 \\
\delta \xi \mathrm{Y} \times 4.593271
\end{array}\right)=24 / 2 \pi \quad\left(\begin{array}{lr}
-24.680 & 6.019 \\
-9.338 & 16.133
\end{array}\right)\left(\begin{array}{l}
\mathrm{K} 2 \mathrm{x} \\
\mathrm{K} 2 \mathrm{y}
\end{array}\right)
\end{aligned}
$$

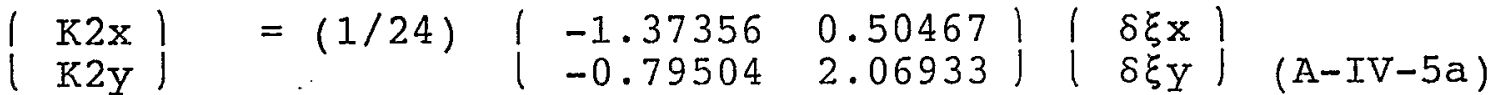

The same matxix calculated by A. Luccio using MAD [54] was

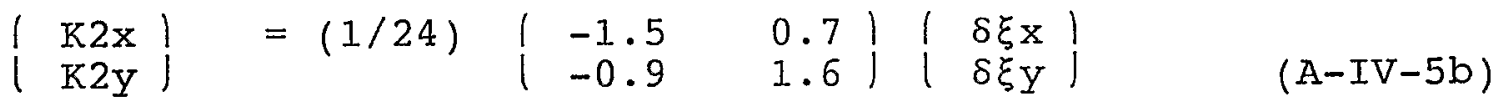

or by $W$. van Asselt

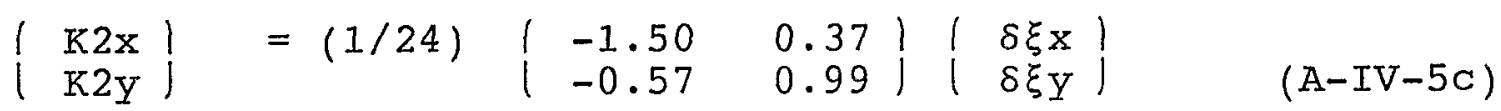

and the measurement by $W$. van Asselt and $L$. Ahrens $[55,56]$ was

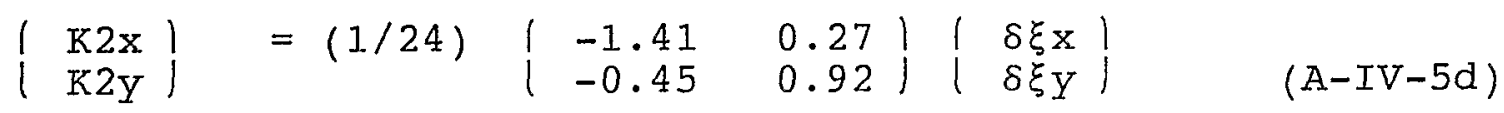

They roughly agree with each other. The strength of the chromaticity sextupoles when the normalized chromaticities were set at $\xi x=-0.50$ and $\xi_{Y}=-0.25$ were calculated using $(A-I V-5 a)$. The accuracy of (A-IV-5a) is maybe the worse among these four equations but we don't have to care.

$$
(1 / 24)\left(\begin{array}{ll}
-1.37356 & 0.50467 \\
-0.79504 & 2.06933
\end{array}\right)\left(\begin{array}{l}
-0.50+1.568 \\
-0.25+0.623
\end{array}\right)=\left(\begin{array}{l}
-0.0533 \\
-0.0032
\end{array}\right) \text {. }
$$

These are comparable to the edge sextupole of the dipole magnet:

$$
(2 \pi / 72) \times 0.24=0.021 \text {. }
$$


Appendix V Eddy-current field correction at C5

The vacuum chamber and the eddy-current correction winding at C5 is not like ones at the other cells. The middle part of the beam duct in the C5 bending dipole is wider than the normal ones and the center is displaced outward in order to inject the proton beam. To cancel the eddy-current field it has two correction windings as schematically shown in Fig.A-2 [57]. One correction winding is winded on up-stream $3 / 4$ of the beam duct to cancel the sextupole component. The other is winded on downstream $1 / 4$ of the beam duct to cancel the quadrupole component. Because the location of the correction windings and the eddy-current field are different, the corrections can not be perfect. In this Appendix $V$ we will estimate this imperfection. The beta functions and betatron phases at the bending magnet at $C 5$ are listed in Table A-VI. The ratio of the correction error to the correction is defined by

$$
\Delta \mathrm{C} 5=\mathrm{I} \text { WC } \mathrm{e}^{\mathrm{jk \theta}}-\text { We } \mathrm{W} / \text { We } . \quad(\mathrm{A}-\mathrm{V}-1)
$$

Here We and Wc are weight functions of resonance at the location of the eddy-current field and at the location of the correction winding. The weight function $\mathrm{W}$ is

$$
\begin{aligned}
& W=\eta^{2} \sqrt{ } x^{m} \sqrt{ } / B y^{n} \\
& \Delta \theta=\theta c-\theta e
\end{aligned}
$$

for the $1-t h$ down-feeding to the resonance $m Q x+n Q y=k$.

About $80 \%$ of the induced dipole eddy-current field is not corrected at the normal cell [38]. The uncorrected dipole field at C5 is almost the same as that of the normal cell [39]. When dipole field is corrected only by the sextupole winding, the difference of locations between the eddy-current field and the correction winding will produce $7 \%$ error of the dipole correction.

The quadrupole field produced by the eddy-current at the center of the vacuum chamber is corrected by the quadrupole correction winding at the downstream end of the vacuum chamber. The error of corrections are 42 and $33 \%$ of corrections for $2 Q x=9$ and $20 y=9$, respectively. That means even if the eddy-current correction system worked as was designed, we expected a normal quadrupole $\mathrm{d} B / d t$ term. The strength of the expected (or designed) $\mathrm{dB} / \mathrm{dt}$ term is about $1 / 6$ of the observed at the present, which would be still considerable.

The error of the sextupole corrections are 18\%, 13\%. 18\% and $9 \%$ for $3 Q x=14, Q x=2 Q Y=14$, down-feeding to $2 Q x=9$ and down-feeding to $2 Q Y=9$, respectively. The strengths of these unavoidable correction errors are comparable to the contributions of down-feeding from the octupole imperfections. 
Table A-VI Twiss parameters at $\mathrm{C} 5$ when $Q \mathrm{X}=4.6337$ and $Q Y=$ 4.5833. $\Delta \theta Y Y x=(2 \Delta \theta y+\Delta \theta x) / 3$.

\begin{tabular}{|c|c|c|c|c|c|c|}
\hline parameter & $\begin{array}{l}\text { upstre } \\
\text { edge }\end{array}$ & $\operatorname{am}_{3 / 8}$ & $\begin{array}{l}\text { middle } \\
1 / 2\end{array}$ & $7 / 8^{\text {dor }}$ & $\begin{array}{l}\text { mstream } \\
\text { end }\end{array}$ & $\begin{array}{c}\text { err.ratio } \\
\triangle \mathrm{C} 5\end{array}$ \\
\hline 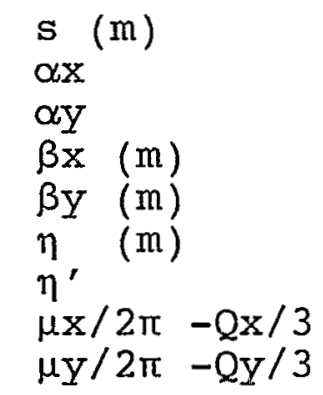 & $\begin{array}{r}18.599 \\
-0.748 \\
1.753 \\
4.803 \\
12.297 \\
1.766 \\
0.233 \\
0.450 \\
0.405\end{array}$ & 19.5065 & $\begin{array}{r}19.809 \\
-1.100 \\
1.355 \\
7.045 \\
8.537 \\
2.094 \\
0.309 \\
0.483 \\
0.424\end{array}$ & 20.7165 & $\begin{array}{r}21.019 \\
-1.420 \\
0.955 \\
10.102 \\
5.742 \\
2.513 \\
0.382 \\
0.506 \\
0.451\end{array}$ & \\
\hline $\begin{array}{l}\beta x(m) \\
\beta y \quad(m) \\
\eta \quad(m) \\
\Delta \theta x \quad \text { (mrad.) } \\
\Delta \theta y \text { (mrad.) }\end{array}$ & & $\begin{array}{l}6.399 \\
9.387 \\
2.003 \\
-9.71 \\
-7.36\end{array}$ & $\begin{array}{l}7.045 \\
8.537 \\
2.094 \\
0 \\
0\end{array}$ & $\begin{array}{c}9.267 \\
6.350 \\
2.497 \\
26.31 \\
26.60\end{array}$ & & \\
\hline $\begin{array}{l}\text { HORIZONTAT D } \\
\sqrt{ } \beta x \cos (4.8 \\
\sqrt{ } \beta x \sin (4.8\end{array}$ & $\begin{array}{l}\text { POIE } \\
\theta x) \\
\theta x)\end{array}$ & $\begin{array}{r}2.527 \\
-0.118\end{array}$ & $\begin{array}{l}2.654 \\
0\end{array}$ & & & 0.0653 \\
\hline $\begin{array}{l}\text { QUADRUPOLE } \\
\beta x \cos (9 \Delta \theta x) \\
\beta x \sin (9 \Delta \theta x\end{array}$ & & & $\begin{array}{l}7.045 \\
0\end{array}$ & $\begin{array}{r}9.00 \\
2.174\end{array}$ & & \\
\hline $\begin{array}{l}\beta y \cos (9 \Delta \theta y) \\
\beta y \sin (9 \Delta \theta y\end{array}$ & & & $\begin{array}{l}8.537 \\
0\end{array}$ & $\begin{array}{r}6.16 \\
1.506\end{array}$ & & \\
\hline
\end{tabular}

\begin{tabular}{|c|c|c|c|}
\hline \multicolumn{4}{|c|}{ QUADRUPOLE DOWN-FEEDING (2ND) } \\
\hline$\eta \beta x \cos (9 \Delta \theta x)$ & 12.768 & 14.752 & \\
\hline & & & 0.1784 \\
\hline$\eta \beta y \cos (9 \Delta \theta y)$ & 18.761 & 17.876 & \\
\hline$\eta \beta y \sin (9 \Delta \theta y)$ & -1.245 & 0 & 0.0854 \\
\hline SEXTUPOLE & & & \\
\hline$\beta x / \beta x \cos (14 \Delta \theta x)$ & 16.038 & 18.699 & \\
\hline$\beta x / \beta x \sin (14 \Delta \theta x)$ & -2.194 & 0 & 0.1844 \\
\hline $\begin{array}{l}\beta y / \beta x \cos (14 \Delta \theta y y x) \\
\beta y / \beta x \sin (14 \Delta \theta y y x)\end{array}$ & $\begin{array}{l}23.591 \\
-2.702\end{array}$ & $\begin{array}{c}22.659 \\
0\end{array}$ & \\
\hline & & & 0.1261 \\
\hline
\end{tabular}


The integrated quadrupole field produced by the eddy-current in the vacuum chamber at C5 was [39]

$$
\delta B 1=50.5 \text { Gauss for } \mathrm{dB} / \mathrm{dt}=80 \mathrm{G} / \mathrm{ms} \text {. }
$$

The induced eddy-current quadrupole field is horizontally defocussing quadrupole when $B$ is increasing. The focusing quadrupole (positive correction) is required to cancel it. So it produces the $B$ terms:

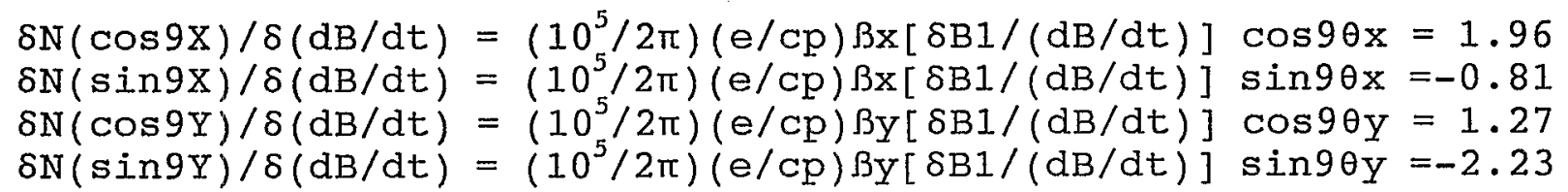
here

$$
\begin{array}{ll}
9 \theta x=-0.389+8 \pi & \text { and } 9 \theta y=-1.052+8 \pi, \\
\beta x=7.045 \mathrm{~m} & \text { and } \beta y=8.537 \mathrm{~m}, \\
\delta \mathrm{B} I /(\mathrm{dB} / \mathrm{dt})=6.31 \mathrm{XI0}-5 & (\mathrm{~T} / \mathrm{m}) /(\mathrm{G} / \mathrm{ms}) .
\end{array}
$$

On the other hand the correction winding produces

$$
\begin{aligned}
& \delta N(\cos 9 X) / \delta(d B / d t)=-2.76 \\
& \delta N(\sin 9 X) / \delta(d B / d t)=0.42 \\
& \delta N(\cos 9 Y) / \delta(d B / d t)=-1.31 \\
& \delta N(\sin 9 Y) / \delta(d B / d t)=1.39
\end{aligned}
$$

here

$$
\begin{array}{ll}
9 \theta x=-0.152+8 \pi & \text { and } 9 \theta y=-0.813+8 \pi \\
\beta x=9.267 \mathrm{~m} & \text { and }
\end{array} \beta_{y=6.350 \mathrm{~m} .}
$$

The integrated sextupole field produced by the eddy-current in the vacuum chamber at C5 was [39]

$$
\delta B 2=3.63 \mathrm{kGauss} / \mathrm{m}^{2} \text { for } \mathrm{dB} / \mathrm{dt}=80 \mathrm{G} / \mathrm{ms} \text {. }
$$

which was smaller than the sextupole correction at the normal cell $[32,38]:$

$$
\delta B 2=5.27 \mathrm{kGauss} / \mathrm{m}^{2} \text { for } \mathrm{dB} / \mathrm{dt}=80 \mathrm{G} / \mathrm{ms} \text {. }
$$

The induced eddy-current sextupole field is horizontally focussing sextupole when $B$ is increasing. The defocussing sextupole (positive correction) is required to cancel it. So it produces the $B$ terms:

$$
\begin{aligned}
& \delta \mathrm{N}(\cos 14 \mathrm{x}) / \delta(\mathrm{dB} / \mathrm{dt})=125(\mathrm{e} / \mathrm{cp}) \beta \mathrm{x} / \beta \mathrm{x}[\delta \mathrm{B} 2 / \delta(\mathrm{dB} / \mathrm{dt})] \cos 14 \theta \mathrm{x} \\
& \delta \mathrm{N}(\sin 14 \mathrm{X}) / \delta(\mathrm{dB} / \mathrm{dt})=125(\mathrm{e} / \mathrm{cp}) \beta \mathrm{x} / \beta \mathrm{x}[\delta \mathrm{B} 2 / \delta(\mathrm{dB} / \mathrm{dt})] \sin 14 \theta \mathrm{x} \\
& \delta \mathrm{N}(\cos 14 \mathrm{XY}) / \delta(\mathrm{dB} / \mathrm{dt})=125(\mathrm{e} / \mathrm{cp}) \beta \mathrm{Y} / \beta \mathrm{x}[\delta \mathrm{B} 2 / \delta(\mathrm{dB} / \mathrm{dt})] \cos 14 \theta \mathrm{xyY} \\
& \delta \mathrm{N}(\sin 14 \mathrm{XY}) / \delta(\mathrm{dB} / \mathrm{dt})=125(\mathrm{e} / \mathrm{cp}) \beta y / \beta x[\delta B 2 / \delta(\mathrm{dB} / \mathrm{dt})] \sin 14 \theta x y y \text {. }
\end{aligned}
$$


The sign of the slope of quadrupole corrections is negative because of the definition of the correction polarity (Appendix II).

$\delta^{2} \mathrm{~N}(\cos 9 \mathrm{X}) / \delta \mathrm{dRset} / \delta(\mathrm{dB} / \mathrm{dt})$

$=-\left(10^{5} / 2 \pi\right)(\mathrm{e} / \mathrm{cp}) 2 \beta x \eta[(\mathrm{dP} / \mathrm{P}) / \mathrm{dRset}][\delta \mathrm{B} 2 /(\mathrm{dB} / \mathrm{dt})] \cos 9 \theta \mathrm{x}$ $\delta^{2} \mathrm{~N}(\sin 9 \mathrm{X}) / \delta \mathrm{dRset} / \delta(\mathrm{dB} / \mathrm{dt})$

$=-\left(10^{5} / 2 \pi\right)(\mathrm{e} / \mathrm{cp}) 2 B \times \eta[(\mathrm{dP} / \mathrm{P}) / \mathrm{dRset}][\delta \mathrm{B} 2 / \delta(\mathrm{dB} / \mathrm{dt})] \sin 9 \theta \mathrm{x}$ $\delta^{2} \mathrm{~N}(\cos 9 \mathrm{Y}) / \delta \mathrm{dRset} / \delta(\mathrm{dB} / \mathrm{dt})$

$=-\left(10^{5} / 2 \pi\right)(\mathrm{e} / \mathrm{cp}) 2 \beta Y \eta[(\mathrm{dP} / \mathrm{P}) / \mathrm{dRset}][\delta \mathrm{B} 2 / \delta(\mathrm{dB} / \mathrm{dt})] \cos 9 \theta \mathrm{Y}$ $\delta^{2} \mathrm{~N}(\sin 9 \mathrm{Y}) / \delta \mathrm{dR}$ set $/ \delta(\mathrm{dB} / \mathrm{dt})$

$=-\left(10^{5} / 2 \pi\right)(\mathrm{e} / \mathrm{cp}) 2 B y \eta[(\mathrm{dP} / \mathrm{P}) / \mathrm{dRset}][\delta \mathrm{B} 2 / \delta(\mathrm{dB} / \mathrm{dt})] \sin 9 \theta \mathrm{y}$

here $(A-V-6)$

$$
\delta \mathrm{B} 2 /(\mathrm{dB} / \mathrm{dt})=4.54 \times 10^{-3}(\mathrm{~T} / \mathrm{m}) /(\mathrm{G} / \mathrm{ms})
$$

The sextupole correction at $\mathrm{C} 5$ is calculated and listed in Table AVII.

Table A-VII sextupole dB/dt correction at C5.

\begin{tabular}{|c|c|c|c|c|}
\hline parametwes & eddy-c & winding & & \\
\hline $\begin{array}{l}9 \theta x-8 \pi \\
9 \theta y-8 \pi \\
14 \theta x-12 \pi \\
14 \theta x y y-12 \pi \\
\beta x \quad(\mathrm{~m}) \\
\beta y \quad(\mathrm{~m}) \\
\eta \quad(\mathrm{m})\end{array}$ & $\begin{array}{r}-0.389 \\
-1.052 \\
0.792 \\
0.104 \\
7.045 \\
8.537 \\
2.094\end{array}$ & $\begin{array}{r}-0.525 \\
-1.155 \\
0.656 \\
-0.010 \\
6.399 \\
9.387 \\
2.003\end{array}$ & & \\
\hline $\begin{array}{l}\quad \operatorname{cbt} \\
\delta N(\cos 14 X) / \delta(d B / d t) \\
\delta N(\sin 14 X) / \delta(d B / d t) \\
\delta N(\cos 14 X Y) / \delta(d B / d t) \\
\delta N(\sin 14 X Y) / \delta(d B / d t) \\
\delta^{2} N(\cos 9 X) / \delta d R s e t / \delta(d B / d t) \\
\delta^{2} N(\sin 9 X) / \delta d R s e t / \delta(d B / d t) \\
\delta^{2} N(\cos 9 Y) / \delta d R s e t / \delta(d B / d t) \\
\delta^{2} N(\sin 9 Y) / \delta d R s e t / \delta(d B / d t)\end{array}$ & $\begin{array}{r}3.24 \\
3.29 \\
5.56 \\
0.58 \\
-1.85 \\
0.76 \\
-1.20 \\
1.75\end{array}$ & $\begin{array}{r}-3.17 \\
-2.44 \\
-5.86 \\
0.06 \\
1.51 \\
-0.87 \\
1.03 \\
-2.33\end{array}$ & $\begin{array}{l}\text { observed } \\
3.49 \pm 0.43 \\
6.00 \pm 0.20 \\
4.74 \pm 0.20 \\
2.64 \pm 0.19 \\
1.06 \pm 0.29 \\
0.45 \pm 0.29 \\
0.94 \pm 0.18 \\
-0.44 \pm 0.06\end{array}$ & $\begin{array}{l}\text { expected } \\
3.42 \\
5.15 \\
5.04 \\
2.00 \\
1.40 \\
0.56 \\
1.11 \\
0.14\end{array}$ \\
\hline
\end{tabular}


FIGURE CAPTIONS

Fig. 1 Example of measured points of stop-bands in the magnet cycle.

Fig.2 Example of plots to determine the correction values.

Fig.3 Programmed excitation functions for the high intensity proton beam. They were calculated from parameters listed in Table I. The calculated correction current of sin14y in (e); 9 th normal sextupole, went over the limit of a power supply from about $35 \mathrm{~ms}$ after T0. So the shown function, which was the operated pattern in the real machine, was a little bit different from the calculated value. The corrections of $2 Q Y=9$ were adjusted later and saved as SHOJI_test2 [16].

(a) main magnet pattern

(b) $2 Q x=9$ and $2 Q Y=9$

(c) $Q x-Q y=0$ and $Q x+Q y=9$

(d) $3 Q x=14$ and $Q x+2 Q Y=14$

(e) $2 \mathrm{QX}+Q \mathrm{QY}=14$ and $2 Q \mathrm{Q}=9$ (slope)

file; newtopI30A70T1.5

file; Bdot_30_70_10

file; Bdot $30^{-} 70^{-} 10$

file; Bdot $30^{-} 70^{-10}$

file; Bdot_30-70-10

Fig.4 Programmed excitation functions for the Gold beam. The patterns were adjusted later for a higher intensity and saved as GoldTunning_Sept25 by K. Zeno [34].

(a) $3 Q x=14$ and $Q x+2 Q y=14$ file; HI_test_func

(b) $2 Q x+Q Y=14$

Fig. 5 Locations of correction parameters in a harmonic phase space.

(a) Co of 9 th normal quadrupole error ( $2 \mathrm{Qx}=9$ and $2 \mathrm{QY}=9$ )

(b) $\mathrm{Cb}$ of 9 th normal quadrupole error ( $2 \mathrm{Qx}=9$ and $2 \mathrm{QY}=9$ )

(c) Cbt of 9 th normal quadrupole error ( $2 \mathrm{Qx}=9$ and $2 \mathrm{QY}=9$ )

(d) Co of skew quadrupole error ( $Q x-Q y=0$ and $Q x+Q y=9$ )

(e) $\mathrm{Cb}$ of skew quadrupole error $(Q x-Q Y=0$ and $Q x+Q Y=9$ )

(f) Cbt of skew quadrupole error ( $Q x-Q Y=0$ and $Q X+Q Y=9$ )

(g) Co of 14 th normal sextupole error ( $3 Q x=14$ and $Q x+2 Q Y=14$ )

(h) $\mathrm{Cb}$ of 14th normal sextupole error $(3 \mathrm{Qx}=14$ and $\mathrm{Qx}+2 \mathrm{QY}=14$ )

(i) Cbt of 14th normal sextupole error ( $3 Q x=14$ and $Q x+2 Q y=14$ )

(j) Co of 14th skew sextupole error $(2 Q \mathrm{Qx}+Q \mathrm{Q}=14)$

(k) $\mathrm{Cb}$ of 14 th skew sextupole error $(2 Q x+Q y=14)$

(1) Cbt of 14th skew sextupole error $(2 Q x+Q y=14)$

(m) Co of 9 th normal sextupole error $(2 \mathrm{QX}=9$ and $2 \mathrm{QY}=9$ )

(n) $\mathrm{Cb}$ of 9 th normal sextupole error $(2 \mathrm{QX}=9$ and $2 \mathrm{QY}=9$ )

(o) Cbt of 9 th normal sextupole error ( $2 Q \mathrm{Q}=9$ and $2 \mathrm{QY}=9$ )

(p) Co of 9 th skew sextupole error $(Q x+Q y=9$ )

(q) $\mathrm{Cb}$ of 9 th skew sextupole error $(Q x+Q Y=9$ )

(r) Cbt of 9 th skew sextupole error ( $Q x+Q y=9$ )

(s) 9 th normal octupole error ( $2 Q x=9$ and $2 Q y=9$ )

Fig.A-1 Expected correlation between corrections of $3 Q x=14$ and $\mathrm{Qx}+2 \mathrm{Qy}=14$. A correction point of $3 \mathrm{Qx}=14$ is at the position indicated by the black spot. The expected region ( $\sigma$ ) for a correction point of $Q X+2 Q Y=14$ is shown by the circle.

Fig.A-2 Schematic view of the vacuum chamber at C5. 


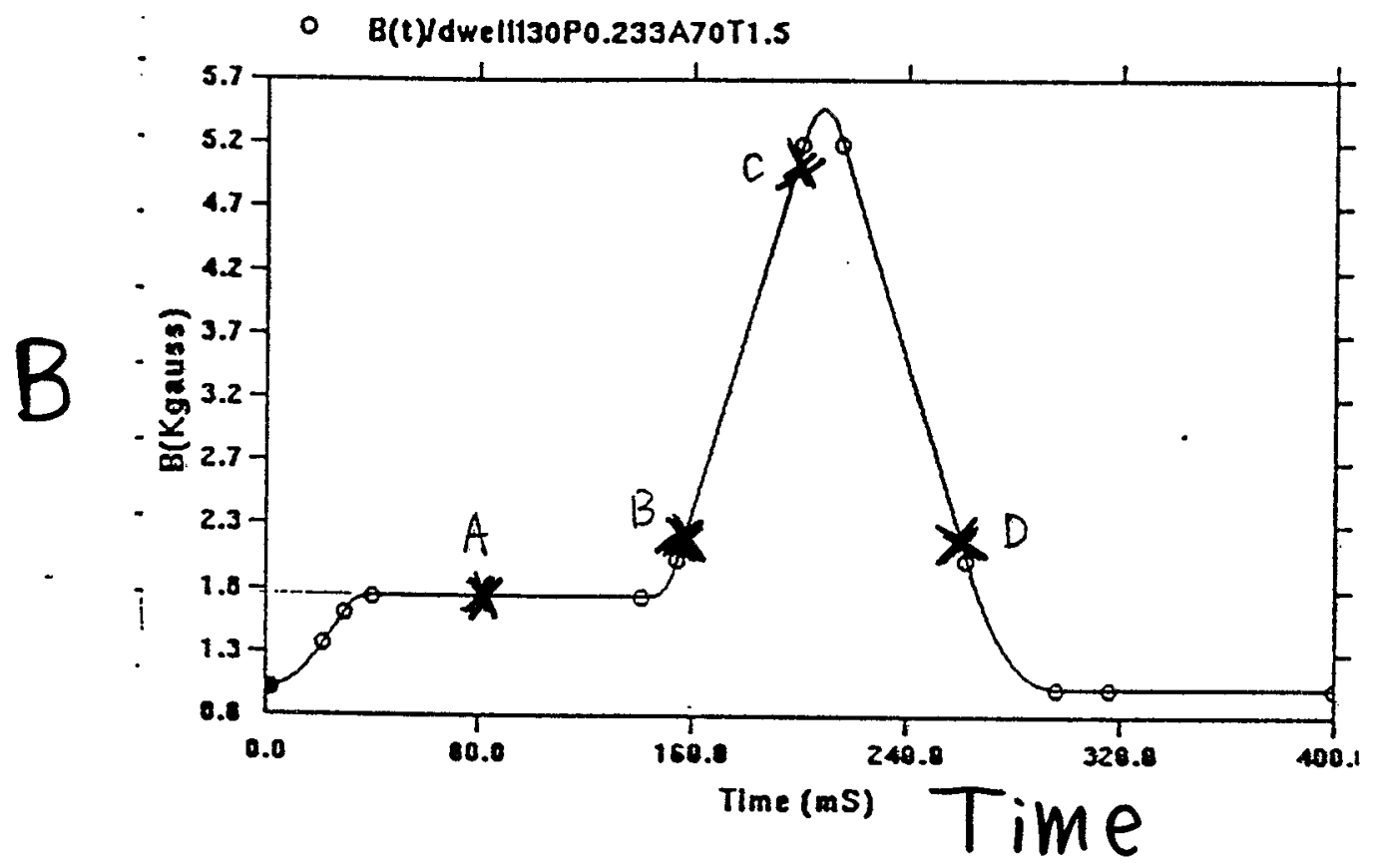

Figure 1 


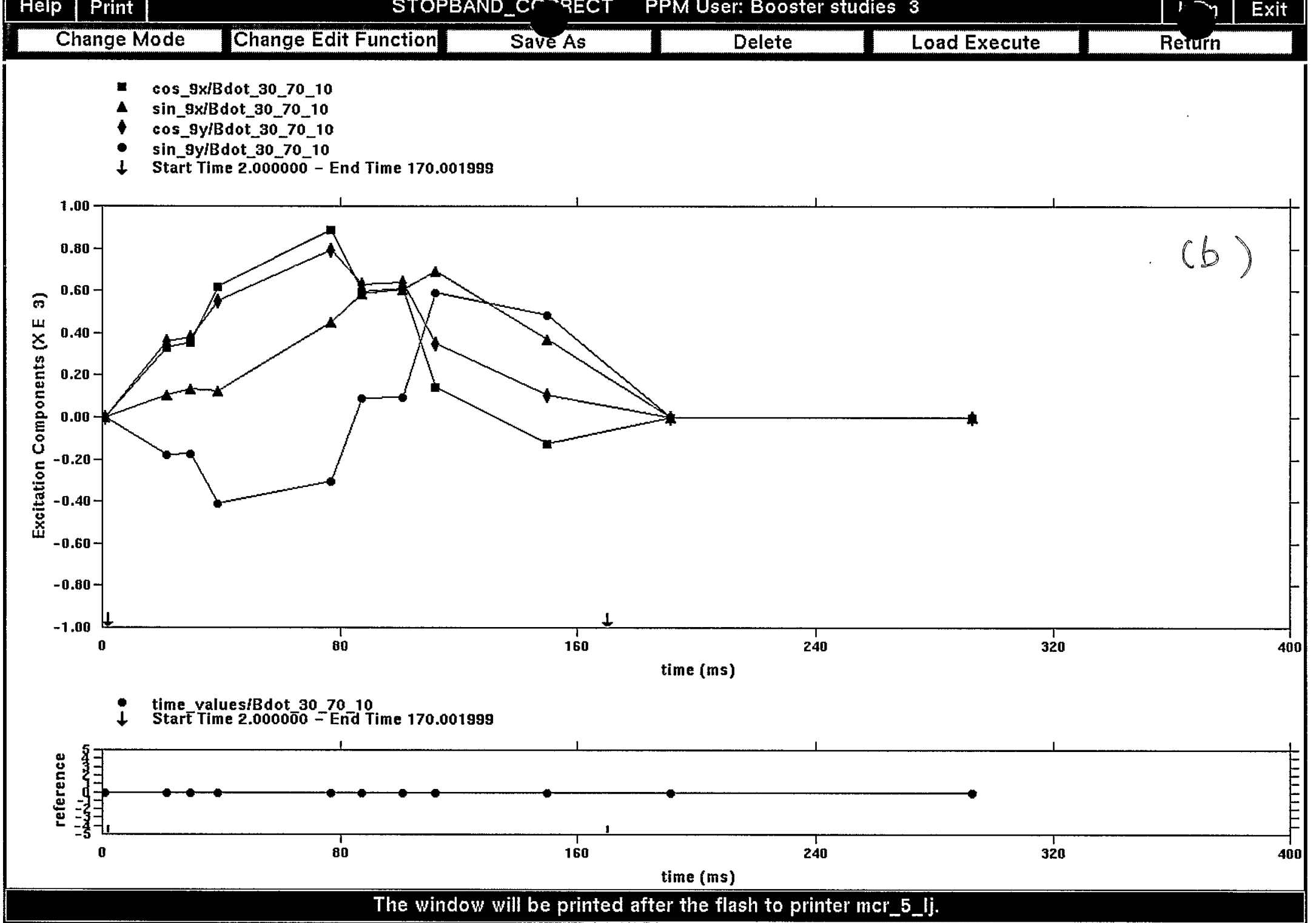




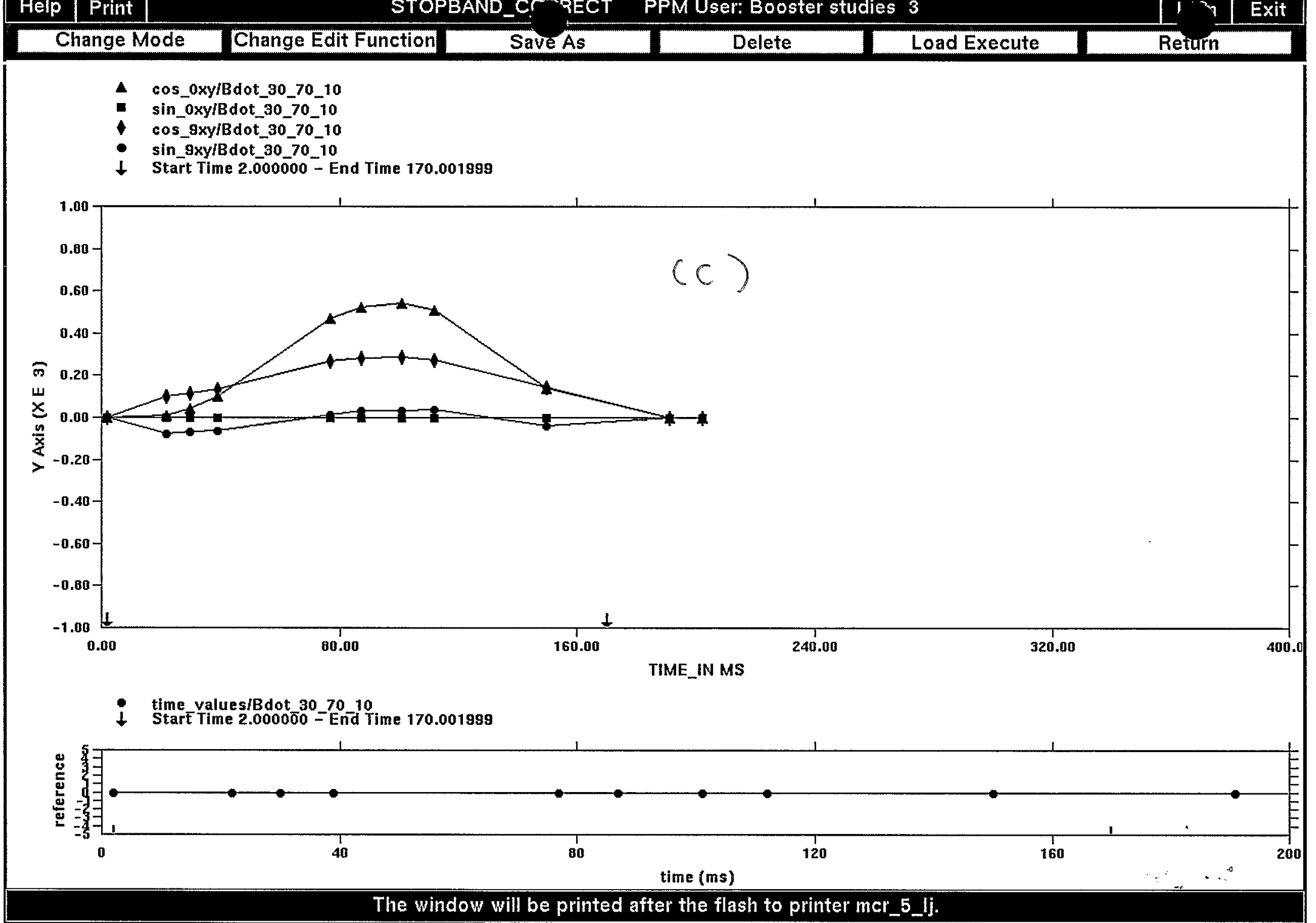

Figure 3 (c) 


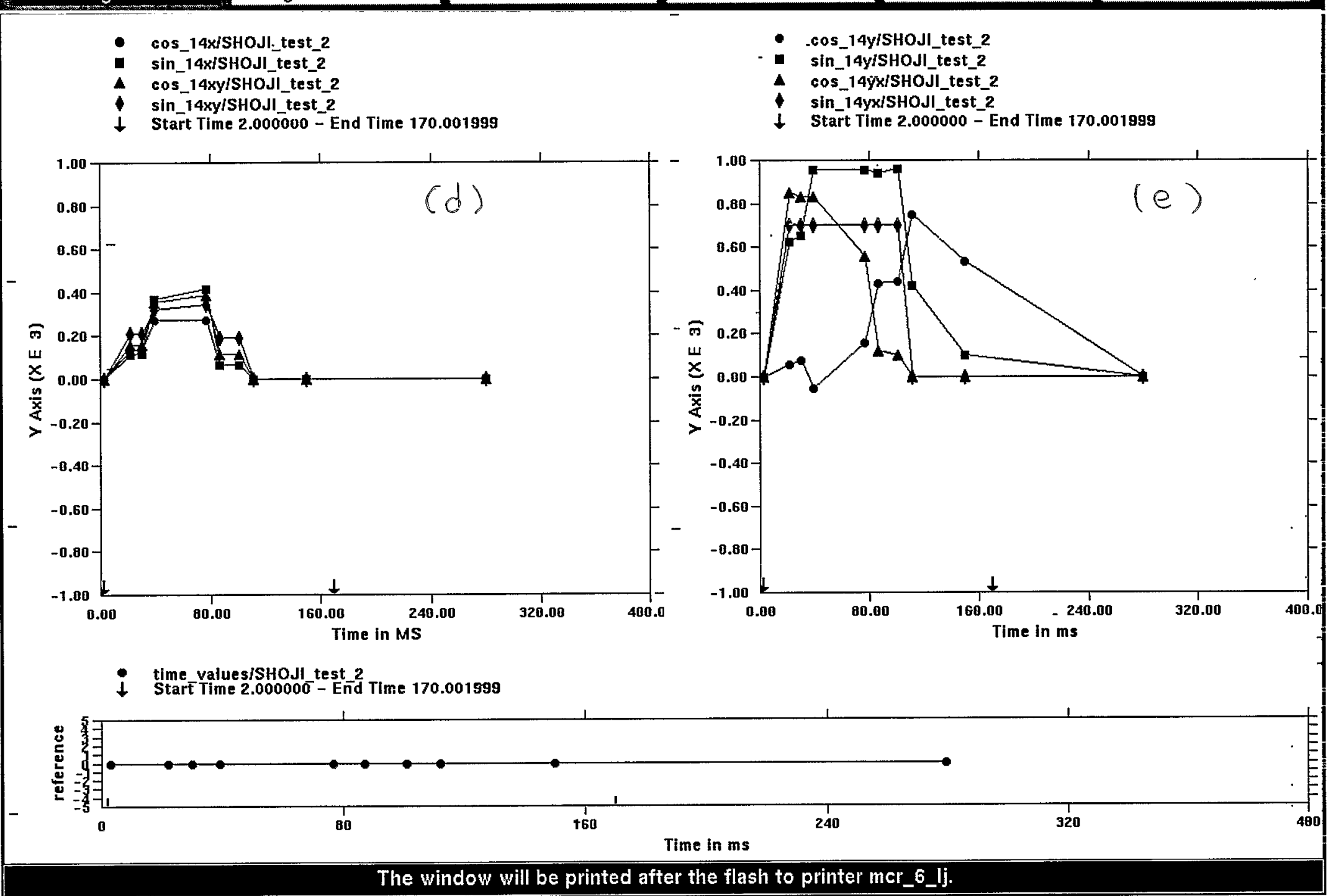




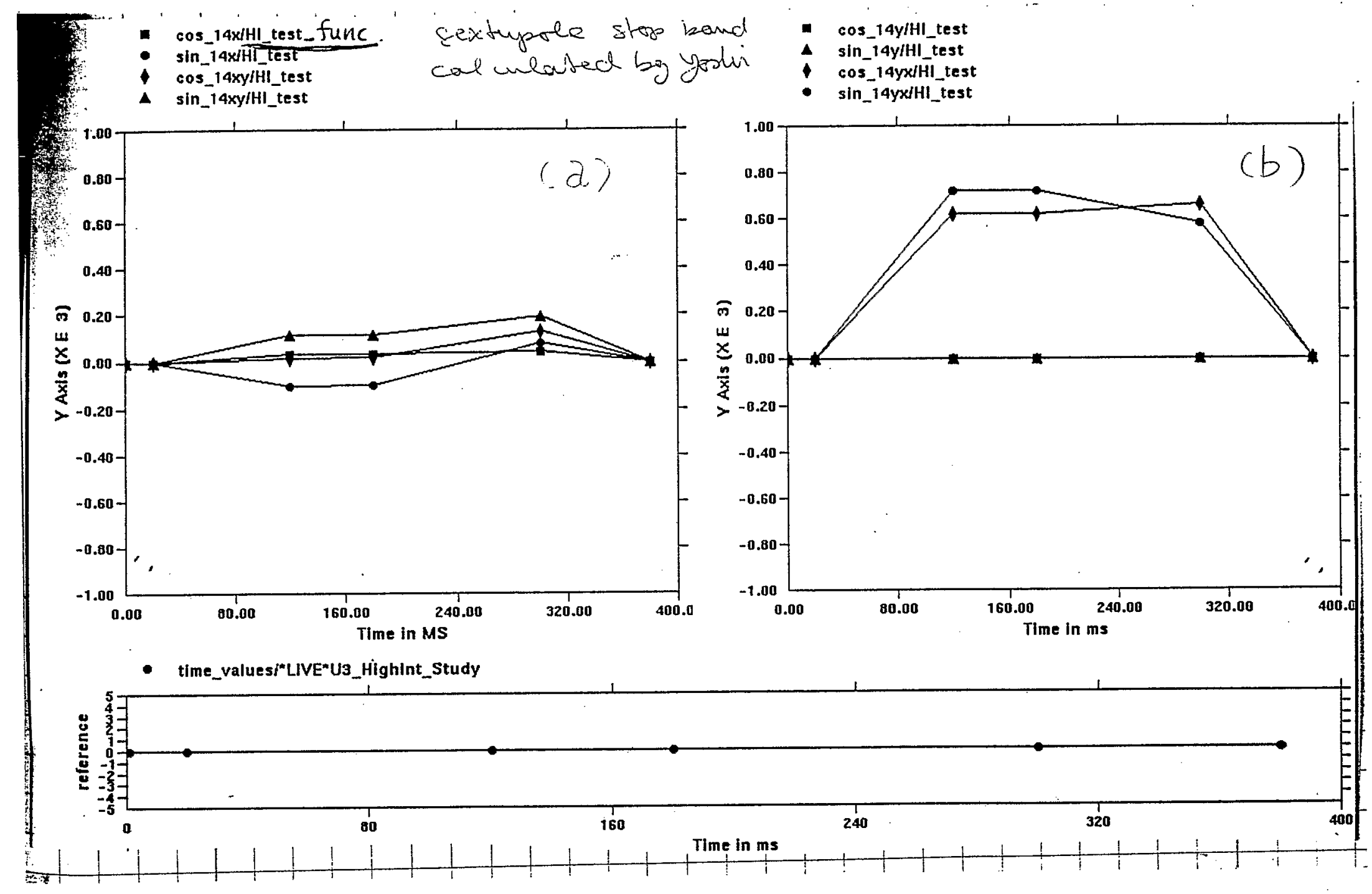

Figure 4 (a) \& (b) 
$-50-$

9 th normal quadrupole 9 th skew quadrupole
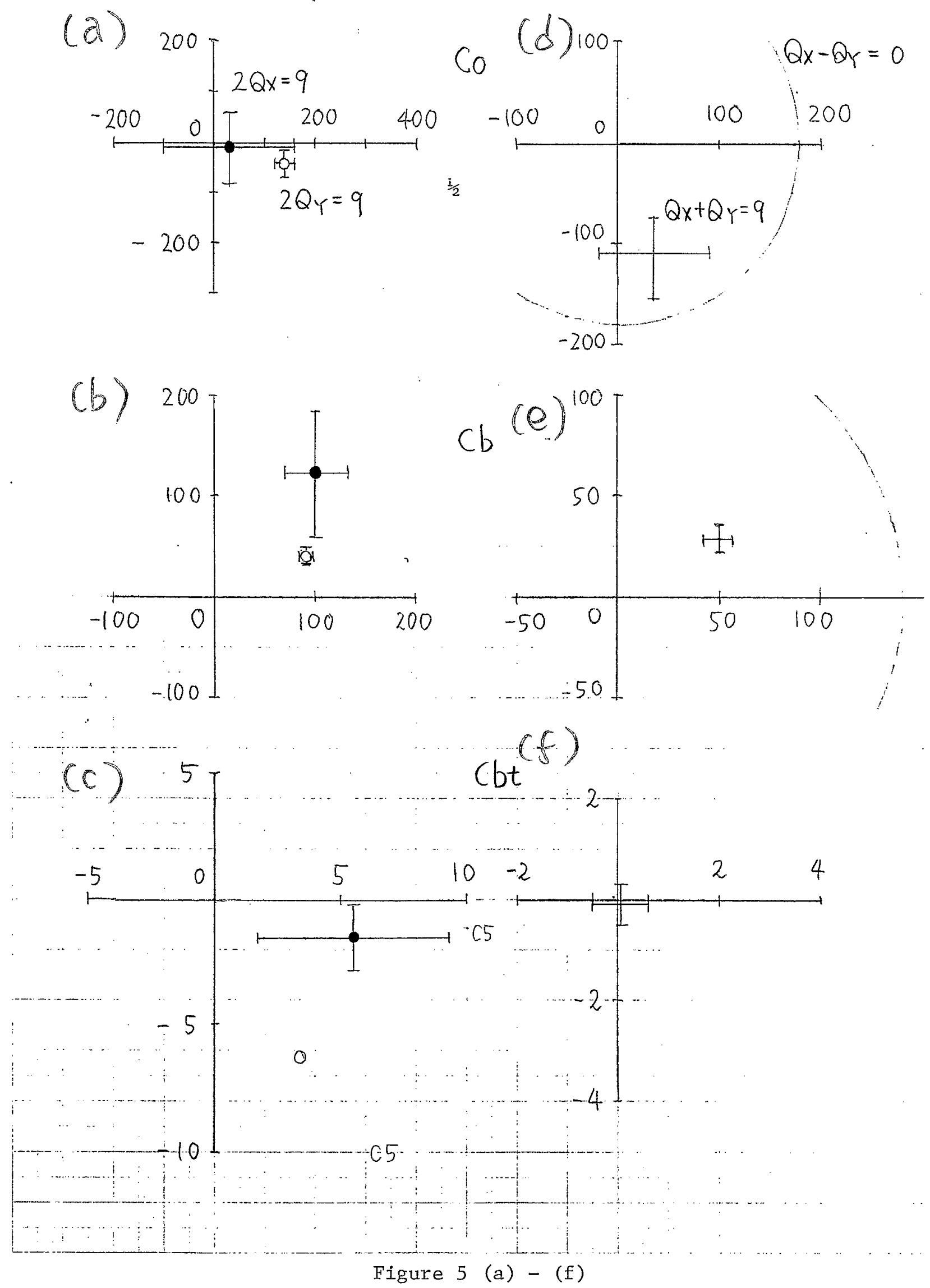


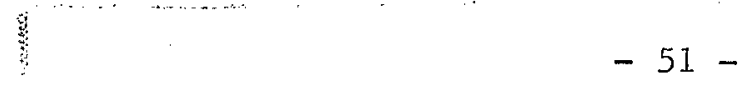

14 th vorital sextupole

$14^{\text {th }}$ skew sextupole

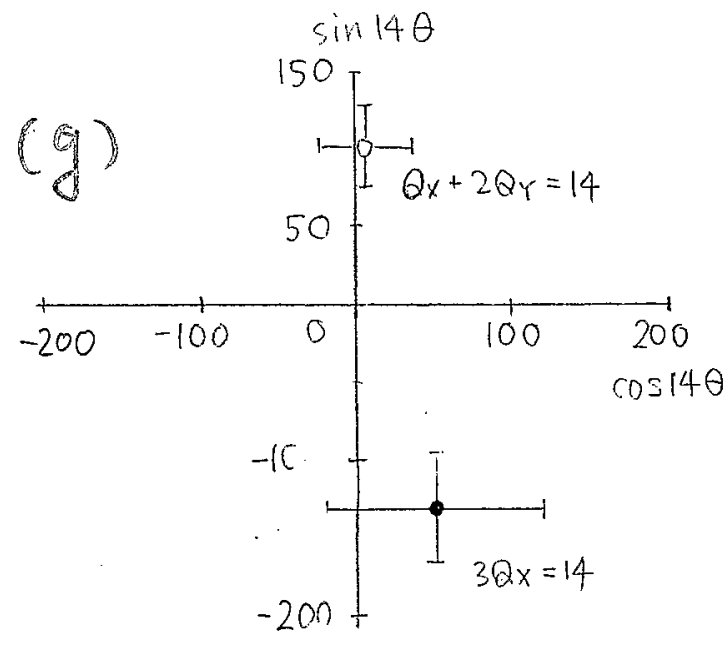

$c_{0}(j)$

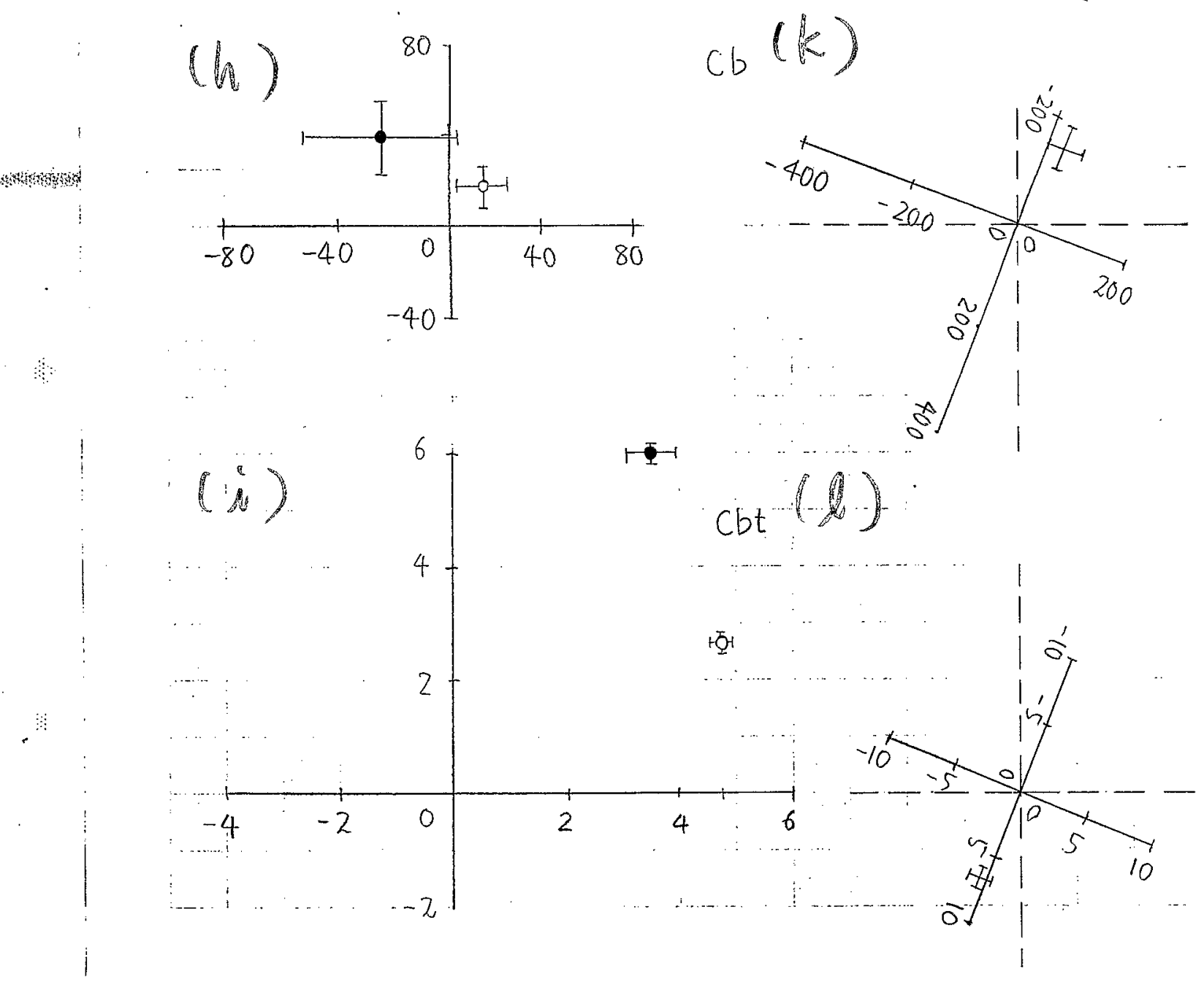

$c b(k)$

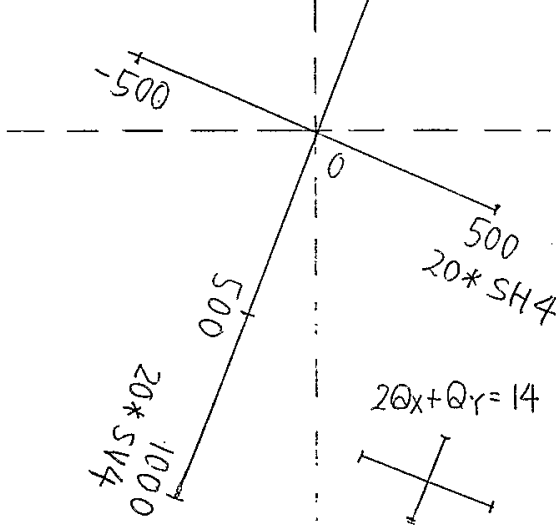

Figure 5 (g) - (1) 
9 th viormal sextupole

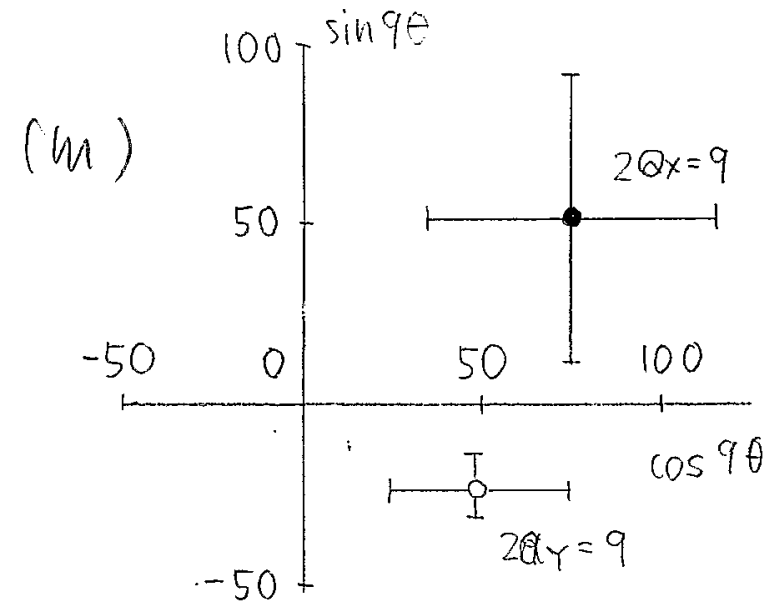

(n)

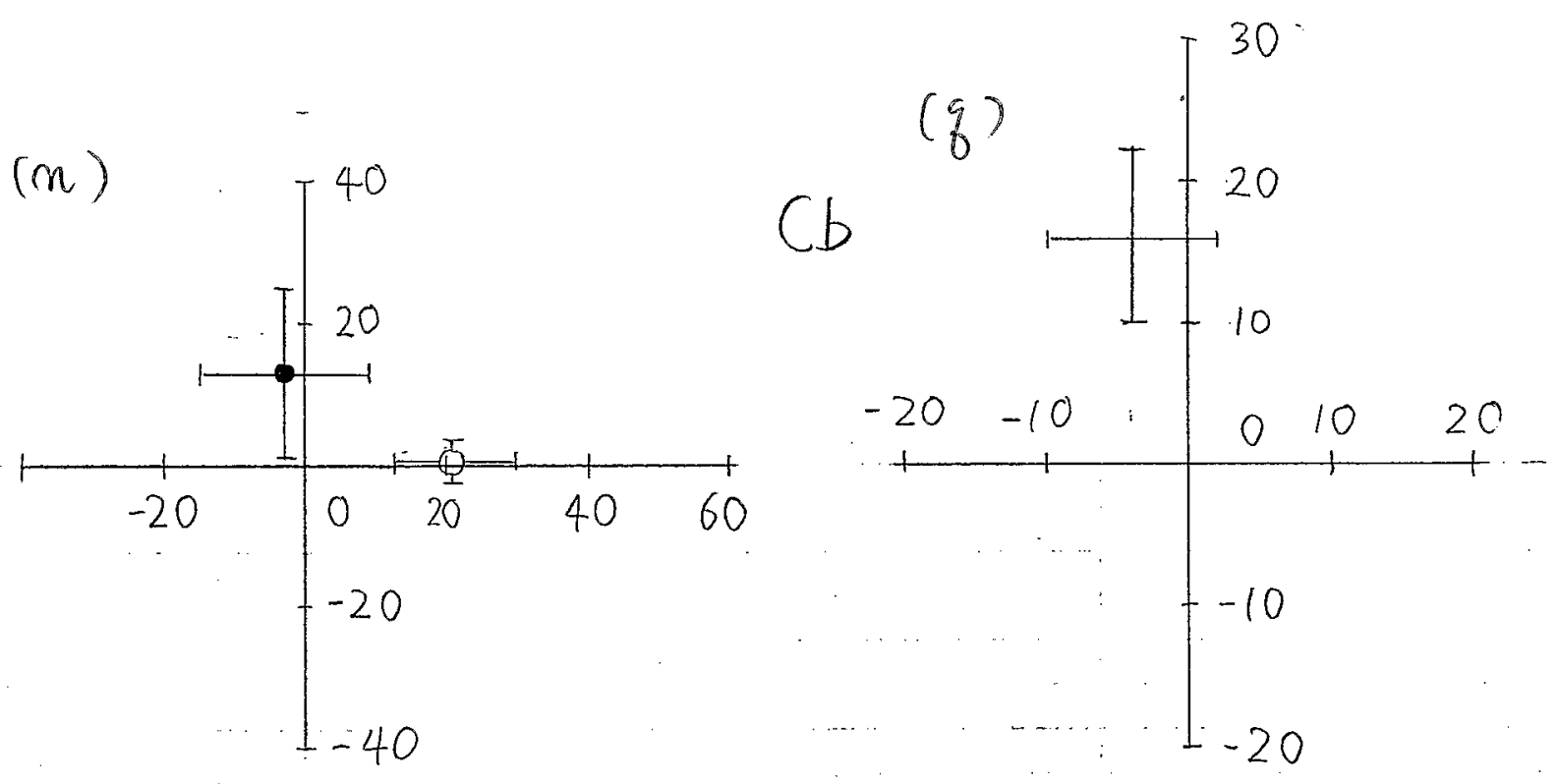

9 th skew sextupole

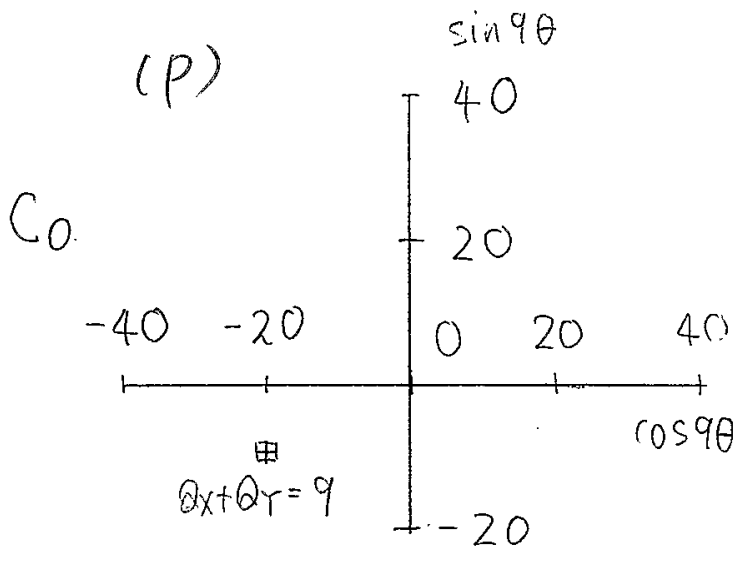

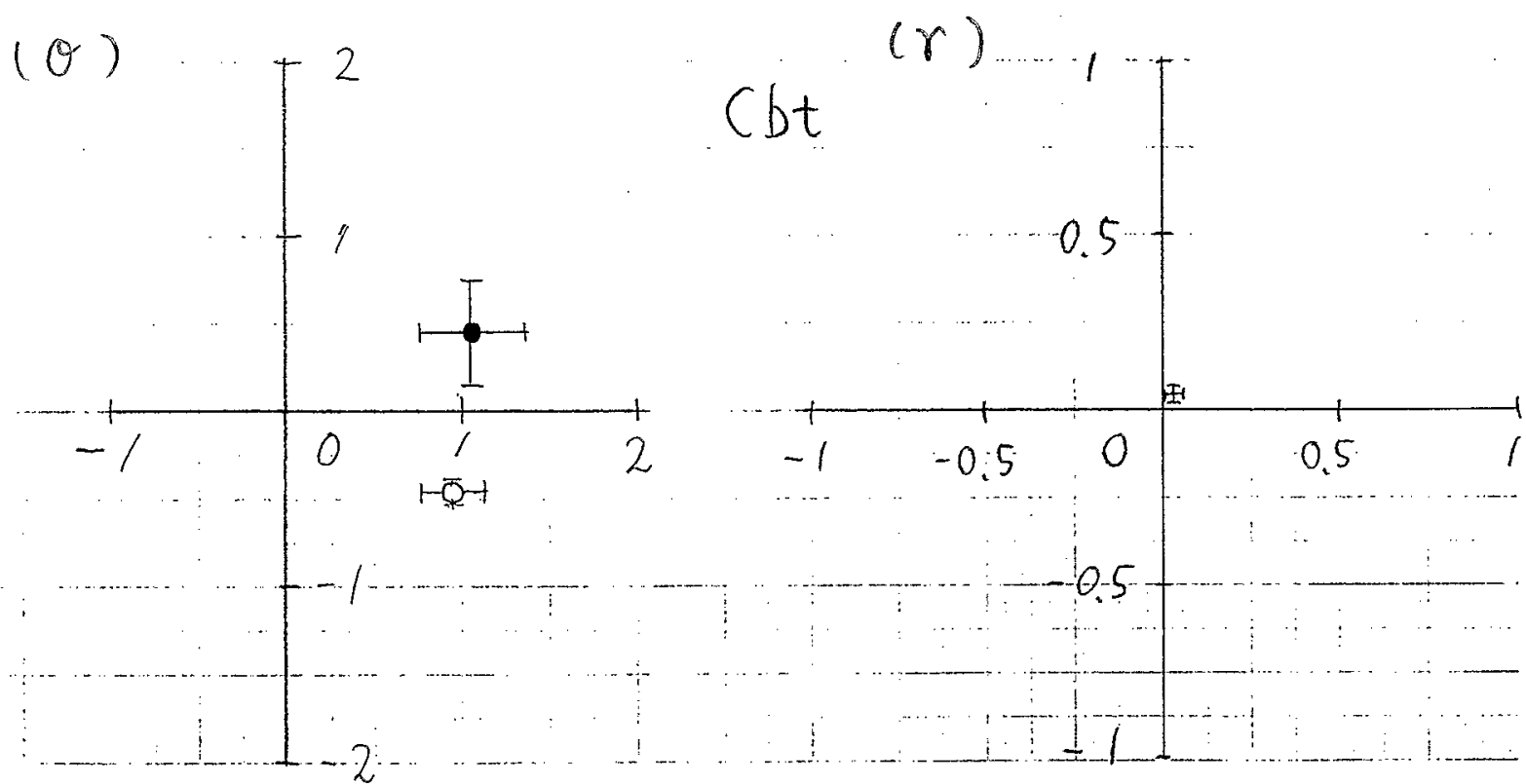

Figure 5 (m) - (r) 
9th normal octupole

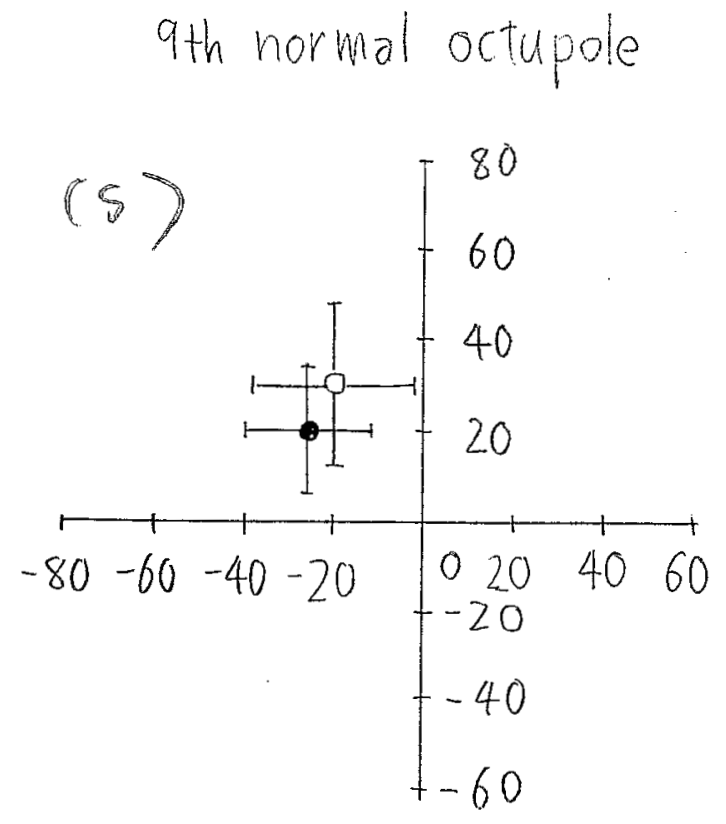

Figure 5 (s)
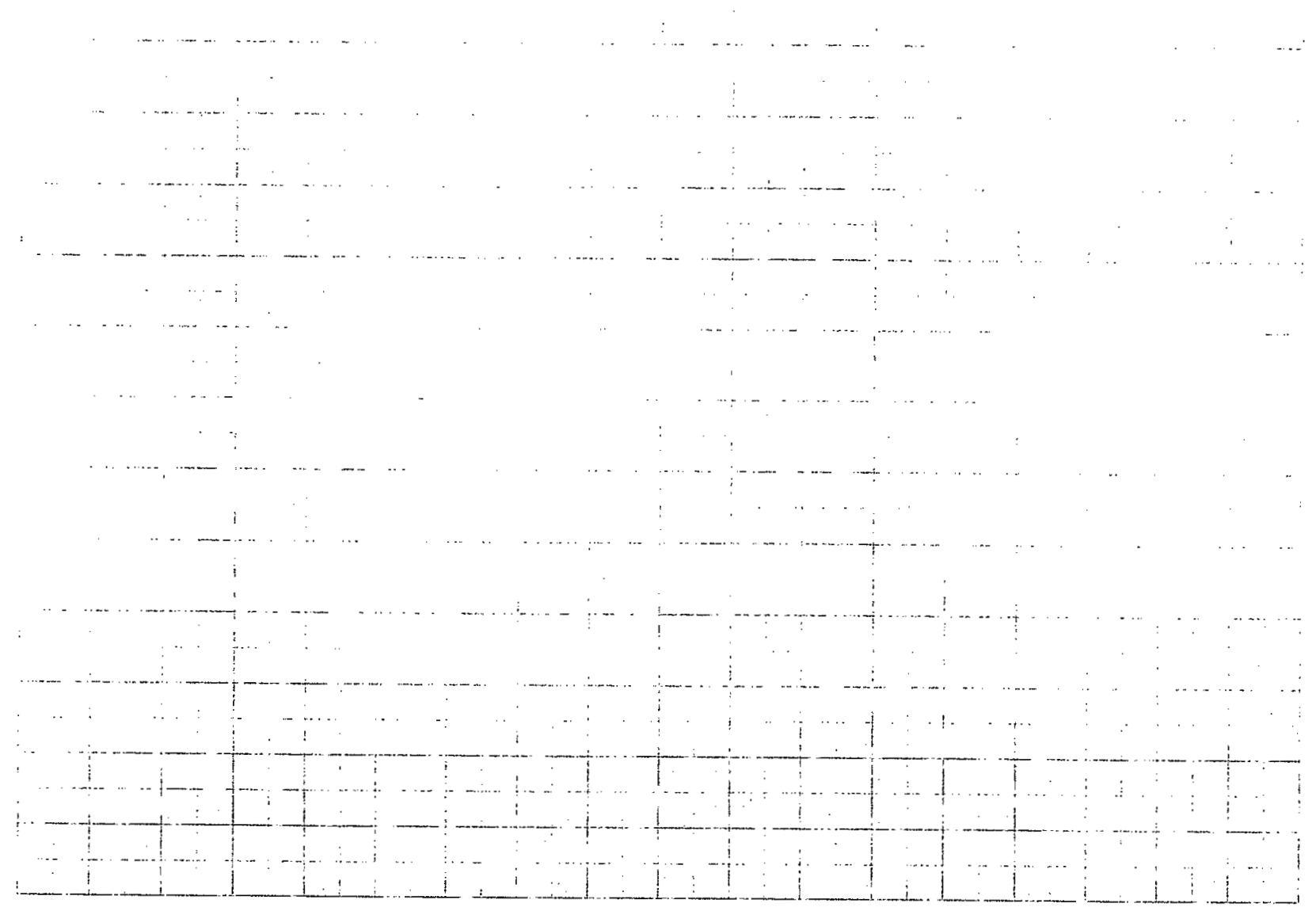


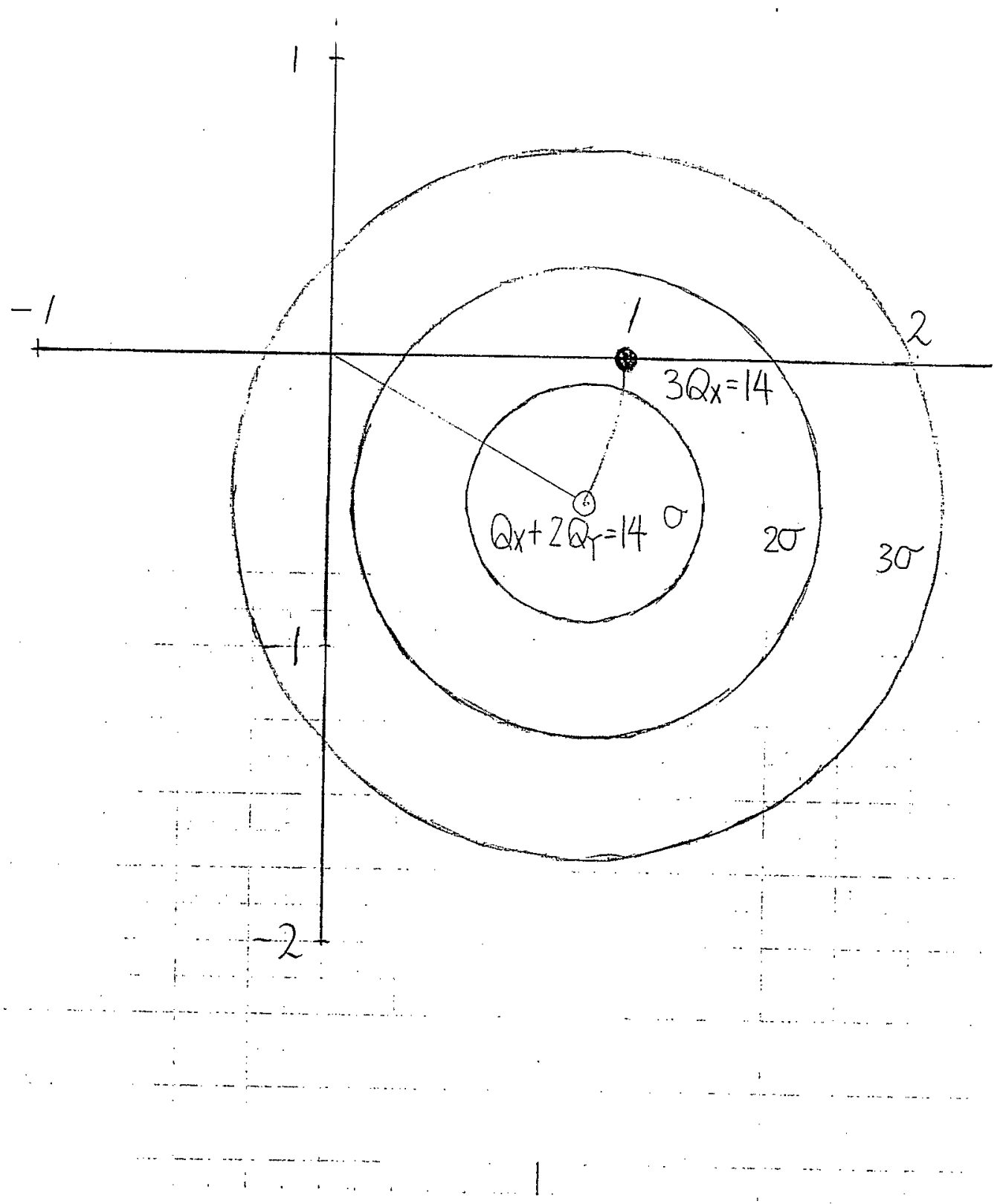

Figure A-1 
$-55-$

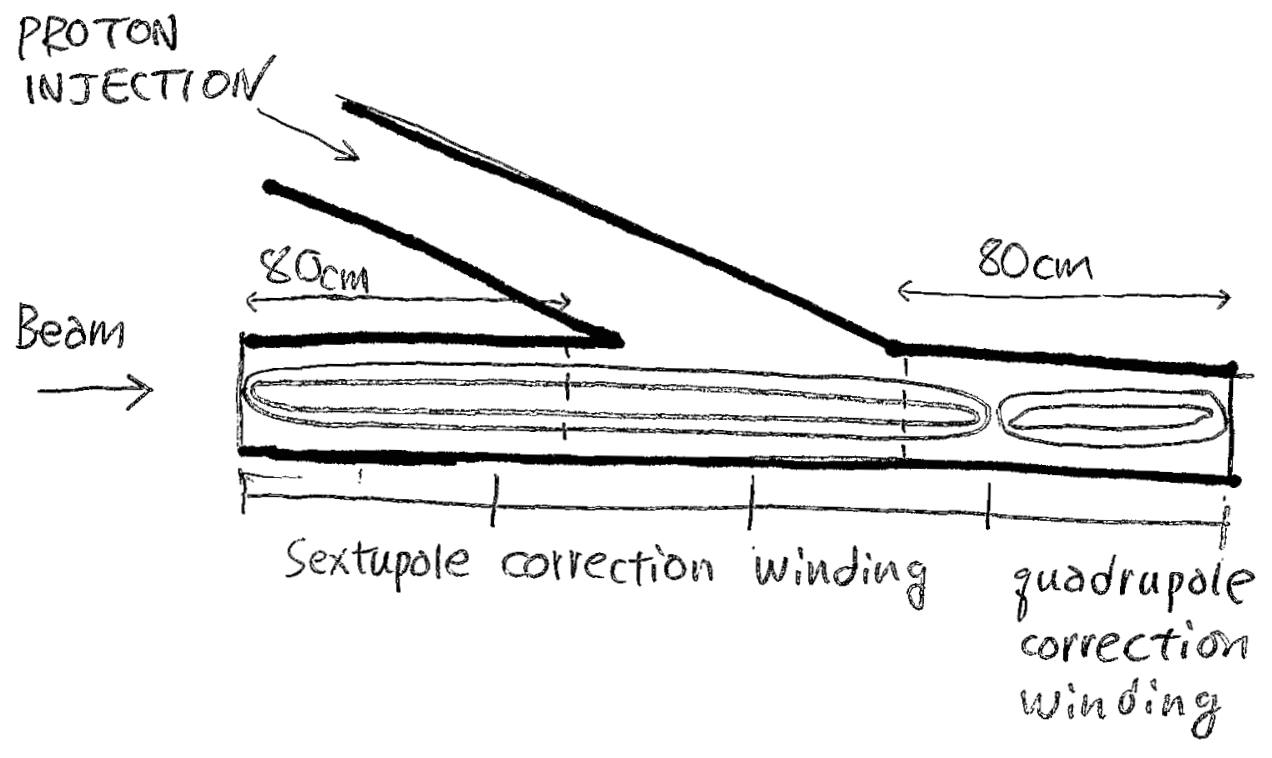

Figure A-2 